The Astrophysical Journal, 671:1748-1765, 2007 December 20

(C) 2007. The American Astronomical Society. All rights reserved. Printed in U.S.A.

\title{
MOLECULAR LINE OBSERVATIONS OF THE SMALL PROTOSTELLAR GROUP L1251B
}

\author{
Jeong-Eun Lee, ${ }^{1,2}$ James Di Francesco, ${ }^{3}$ Tyler L. Bourke, ${ }^{4}$ Neal J. Evans II ${ }^{5}$ and Jingwen Wu ${ }^{4}$ \\ Received 2006 October 11; accepted 2007 August 11
}

\begin{abstract}
We present molecular line observations of L1251B, a small group of pre- and protostellar objects, and its immediate environment in the dense $\mathrm{C}^{18} \mathrm{O}$ core L1251E. These data are complementary to near-infrared, submillimeter, and millimeter continuum observations reported by Lee et al. The single-dish data of L1251B described here show very complex kinematics, including infall, rotation, and outflow motions, and the interferometer data reveal these in greater detail. Interferometer data of $\mathrm{N}_{2} \mathrm{H}^{+} 1-0$ suggest a very rapidly rotating flattened envelope between two young stellar objects, IRS1 and IRS2. In addition, interferometer data of CO 2-1 resolve the outflow associated with L1251B seen in single-dish maps into a few narrow and compact components. Furthermore, the high-resolution data support recent theoretical studies of molecular depletions and enhancements that accompany the formation of protostars within dense cores. Beyond L1251B, single-dish data are also presented of a dense core located $\sim 150^{\prime \prime}$ to the east that Lee et al. detected at $850 \mu \mathrm{m}$, but that has no associated point sources at near- and mid-infrared wavelengths. The relative brightness between molecules, which have different chemical timescales, suggests that it is less chemically evolved than $\mathrm{L} 1251 \mathrm{~B}$. This core may be a site for future star formation, however, since line profiles of $\mathrm{HCO}^{+}, \mathrm{CS}$, and $\mathrm{HCN}$ show asymmetry with a stronger blue peak, which is interpreted as an infall signature.
\end{abstract}

Subject headings: ISM: abundances — ISM: individual (L1251B) — ISM: kinematics and dynamics — ISM: molecules — stars: formation

\section{INTRODUCTION}

Most young stars in our Galaxy formed within groups or clusters (Lada \& Lada 2003). Small, nearby groups of protostellar objects provide compelling cases for investigating the physical processes involved with such formation since the numbers involved are relatively small (e.g., $N<10$ ) and the interactions of these objects with themselves and their environments are less complicated. For example, observational study of kinematics associated with young stars within groups and their immediate dense gas surroundings could provide evidence for a specific formation scenario, for example fragmentation or triggering, that is easier to see because of the simpler nature of groups relative to clusters.

Kinematic study of the gas surroundings of protostellar objects is achieved through observation and interpretation of molecular rotational transitions, since line profiles can be shaped by the motions within the source gas (see Di Francesco et al. 2007 for a review). For example, outflow motions from young stellar objects (YSOs) were first seen in the low-intensity wings of CO 1-0 emission (e.g., Snell et al. 1980). In addition, rotational motions in dense cores have been suggested from the detection of the shift of centroid velocities along different lines of sight in optically thin molecular transitions (e.g., Goodman et al. 1993). Finally, infall motions in dense cores have been the interpretation

\footnotetext{
1 Department of Astronomy and Space Science, Sejong University, Seoul 143-747, Korea.

2 Hubble Fellow, Physics and Astronomy Department, University of California at Los Angeles, Physics and Astronomy Building, 430 Portola Plaza, Box 951547, Los Angeles, CA 90095-1547; jelee@astro.ucla.edu.

3 National Research Council of Canada, Herzberg Institute of Astrophysics, 5071 West Saanich Road, Victoria, BC V9E 2E7, Canada; james.difrancesco@ nrc-cnrc.gc.ca.

4 Smithsonian Astrophysical Observatory, 60 Garden Street, Cambridge, MA 02138; tbourke@cfa.harvard.edu.

5 Astronomy Department, University of Texas at Austin, 1 University Station C1400, Austin, TX 78712-0259; nje@astro.as.utexas.edu, jingwen@astro.as.utexas .edu.
}

of profiles of moderately optically thick lines that are asymmetric with a stronger blue peak relative to optically thin lines (e.g., Zhou et al. 1993; Myers et al. 2000). Such kinematic evidence has come primarily from single-dish telescopes, i.e., of relatively low spatial resolution, in studies of isolated protostellar objects. For protostellar groups, however, the surface density of objects can be high, and single-dish telescopes may not have the resolution needed to associate kinematics with particular protostars within the group. Instead, data from interferometers are very highly suited for such studies, especially when used in tandem with data from singledish telescopes.

The use of molecular lines to study kinematics is complicated by potentially significant molecular abundance variations along the lines of sight toward dense gas both before and after star formation. Such abundance changes can be due to chemical evolution, intertwined with the YSO luminosity evolution, since luminosity affects the temperatures of nearby dust grains that interact with gaseous molecules (see Rawlings \& Yates 2001; Doty et al. 2002; Lee et al. 2004). Of course, YSO luminosity evolution is itself intertwined with dynamical evolution of the surrounding gas, as much of the luminosity originates from accretion. Hence, a holistic approach in general is required, which includes luminosity, dynamics, and chemistry, to best understand the environments from which stars form (see Bergin \& Langer [1997] and Aikawa et al. [2001] for early examples of such models).

Located at $300 \pm 50$ pc (Kun \& Prusti 1993), L1251B is an excellent example of a small, nearby group of protostellar objects where molecular line observations can probe the kinematics and chemistry of its environment and yield clues about its origins. L1251B is located within L1251E, the densest $\mathrm{C}^{18} \mathrm{O}$ core in L1251 (Sato et al. 1994). L1251B is associated with a single IRAS point source, IRAS $22376+7455$, and was found to be associated with CO outflow by Sato \& Fukui (1989). In Lee et al. (2006, hereafter Paper I), we showed via single-dish data that IRAS 22376+ 7455 was associated with a local maximum of submillimeter emission, although significant peaks of $850 \mu \mathrm{m}$ emission were also seen $\sim 150^{\prime \prime}$ to the east. In addition, we showed Spitzer Space Telescope 
TABLE 1

Molecular Line Observations with Single-dish Telescopes

\begin{tabular}{|c|c|c|c|c|c|c|}
\hline Line & $\begin{array}{c}\nu \\
(\mathrm{GHz})\end{array}$ & $\begin{array}{c}\delta v \\
\left(\mathrm{~km} \mathrm{~s}^{-1}\right)\end{array}$ & $\begin{array}{c}\theta_{\mathrm{mb}} \\
(\operatorname{arcsec})\end{array}$ & $\eta_{\mathrm{mb}}^{\mathrm{a}}$ & Observing Dates & Observatory \\
\hline $\mathrm{H}^{13} \mathrm{CO}^{+} 1-0 \ldots \ldots \ldots \ldots \ldots \ldots \ldots \ldots \ldots \ldots \ldots \ldots$ & 86.754285 & 0.14 & 62 & 0.5 & 1997 Apr, 2000 May & FCRAO \\
\hline 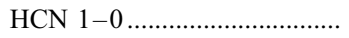 & 88.631847 & 0.10 & 61 & 0.5 & $2005 \mathrm{Feb}$ & FCRAO \\
\hline 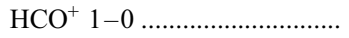 & 89.188523 & 0.13 & 60 & 0.5 & 1997 Apr, 2000 May & FCRAO \\
\hline 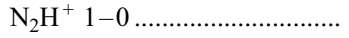 & 93.176265 & 0.13 & 58 & 0.5 & $1996 \mathrm{Dec}$ & FCRAO \\
\hline 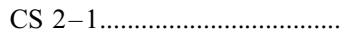 & 97.980968 & 0.10 & 55 & 0.5 & $2005 \mathrm{Feb}$ & FCRAO \\
\hline 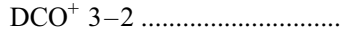 & 216.112605 & 0.13 & 34.7 & 0.74 & $2003 \mathrm{Jul}$ & $\mathrm{CSO}$ \\
\hline $\mathrm{CO} 2-1$ & 230.53800 & 0.12 & 32.5 & 0.64 & $2003 \mathrm{Jul}$ & $\mathrm{CSO}$ \\
\hline $\mathrm{HCO}^{+} 3-2 \ldots \ldots \ldots \ldots \ldots \ldots \ldots$ & 267.557526 & 0.12 & 22.5 & 0.67 & 1997 Jun, 1998 Jul & $\mathrm{CSO}$ \\
\hline CS $3-2 \ldots \ldots$ & 146.969048 & 0.10 & 43 & $0.8^{\mathrm{b}}$ & 1997 Oct & NRAO \\
\hline CS 5-4 & 244.935606 & 0.12 & 26 & $0.5^{\mathrm{b}}$ & 1996 May & NRAO \\
\hline
\end{tabular}

\footnotetext{
a This main beam efficiency is used to convert the antenna temperature $\left(T_{a}^{*}\right)$, only corrected for atmospheric attenuation, radiative loss, and reward scattering and spillover, to the radiation temperature.

$\mathrm{b}$ These numbers represent the corrected main beam efficiencies $\left(\eta_{m}^{*}\right)$ to convert the antenna temperature $\left(T_{R}^{*}\right)$, corrected for forward scattering and spillover as well as atmospheric attenuation, radiative loss, and reward scattering and spillover, to the radiation temperature.
}

observations of the region that revealed $\sim 20$ YSOs within $\sim 1 \mathrm{pc}^{2}$ covering L1251B and its surroundings, indicative of the formation of a group of low-mass stars. Within L1251B itself, these data revealed three Class 0/I objects (IRS1, IRS2, and IRS4) and three Class II objects (IRS3, IRS5, and IRS6), within a projected diameter of 55". (No near- or mid-infrared sources were found to be associated with other locations of submillimeter emission adjacent to L1251B.) Finally, we showed Submillimeter Array (SMA) continuum observations of L1251B in Paper I that revealed two compact locations of high column density between IRS1 and IRS2 that may be starless condensations. Paper I clearly revealed L1251B as a region of recent (and possibly continuing) star formation within a small group.

In this paper, we examine line emission associated with L1251B and the core $\sim 150^{\prime \prime}$ to its east (henceforth, the east core), to probe kinematic and chemical processes in the region. The three embedded Class 0/I sources and two starless objects within L1251B are especially emphasized; IRS1 is the most luminous object, IRS2 is located southeast of IRS1 and is associated with a near-infrared bipolar nebula, and IRS4 is located at the west of IRS1. The two starless objects are named SMA-N (the northern one) and SMA-S (the southern one) in this paper. Besides the outflow noted by Sato \& Fukui, earlier molecular line observations have also provided evidence for rotational and infall motions in L1251B. For example, a velocity gradient of $\sim 2.3 \mathrm{~km} \mathrm{~s}^{-1} \mathrm{pc}^{-1}$ in the northeast-southwest direction across L1251B suggestive of rotational motion was detected by Goodman et al. (1993) in $\mathrm{NH}_{3}$ $(1,1)$ (also Sato et al. 1994; Tóth \& Walmsley 1996; Anglada et al. 1997; Morata et al. 1997). In addition, infall motions toward L1251B were suggested from the detections of asymmetric profiles with stronger blue peaks in $\mathrm{HCO}^{+} 1-0$ and $3-2, \mathrm{CS} 2-1$, and $\mathrm{H}_{2} \mathrm{CO} 2_{12}-1_{11}$ by Gregersen et al. (2000) and Mardones et al. (1997), although observations were published only toward the IRAS point source position. Since the relative intensity of a given molecular line depends on distributions of density, temperature, and molecular abundance along the line of sight, many other transitions and positions needed to be observed within L1251B and its surroundings to correctly understand their physical conditions and dynamical processes. Here, we have gathered with single-dish telescopes or interferometers new observations of L1251B in CS $2-1,3-2$, and 5-4, HCN $1-0, \mathrm{CO} 2-1,{ }^{13} \mathrm{CO}$ $2-1, \mathrm{C}^{18} \mathrm{O} 2-1, \mathrm{HCO}^{+} 1-0$ and $3-2, \mathrm{H}^{13} \mathrm{CO}^{+} 1-0, \mathrm{DCO}^{+} 3-2$, $\mathrm{N}_{2} \mathrm{H}^{+} 1-0$, and $\mathrm{H}_{2} \mathrm{CO}_{12}-2_{11}$.
Details of the acquisition of observations of L1251B for this paper are summarized in $\S 2$. Results and analysis of the data are described in $\S 3$ and $\S 4$. In $\S 5$, we discuss the chemical and dynamical conditions in L1251B and the east core. Finally, a summary of the conclusions of this paper appears in $\S 6$.

\section{OBSERVATIONS}

\subsection{The Five College Radio Astronomy Observatory (FCRAO) Observations}

Observations of $\mathrm{N}_{2} \mathrm{H}^{+} 1-0$ toward L1251B were made in 1996 December with the FCRAO $14 \mathrm{~m}$ telescope near New Salem, MA, using QUARRY, the 15 element focal plane heterodyne receiver array. Observations of $\mathrm{HCO}^{+} 1-0$ and $\mathrm{H}^{13} \mathrm{CO}^{+} 1-0$ were also made toward L1251B at FCRAO in 1997 April and 2000 May, using the QUARRY (1997) and the 32 element SEQUOIA (2000) focal plane arrays, respectively. The autocorrelator spectrometer was configured with a bandwidth of $20 \mathrm{MHz}$ over 1024 channels, providing a channel separation of $\sim 20 \mathrm{kHz}\left(\sim 0.07 \mathrm{~km} \mathrm{~s}^{-1}\right)$. Typical system temperatures were 500-700 K (QUARRY) and 200-250 K (SEQUOIA). Observations of HCN $J=1-0$ and CS 2-1 toward L1251B were also made at FCRAO in 2005 February in OTF mode, using SEQUOIA with a system temperature of $\sim 120 \mathrm{~K}$. A velocity resolution of $0.1 \mathrm{~km} \mathrm{~s}^{-1}$ was achieved with the $25 \mathrm{MHz}$ bandwidth on the dual channel correlator and the data were acquired in frequency-switching mode with an $8 \mathrm{MHz}$ throw. The average pointing accuracy was about 4.5". Table 1 summarizes observational details of the FCRAO data, including the frequency $(\nu)$, velocity resolution $(\delta v)$, the FWHM beam size $\left(\theta_{\mathrm{mb}}\right)$, the main beam efficiency $\left(\eta_{\mathrm{mb}}\right)$, and the observing date for each line.

\subsection{The Caltech Submillimeter Observatory (CSO) Observations}

We observed the $\mathrm{HCO}^{+} 3-2$, $\mathrm{CO} 2-1$, and $\mathrm{DCO}^{+} 3-2$ lines with the $10.4 \mathrm{~m}$ telescope of the CSO at Mauna Kea, HI, in 1997 June and 2003 July. $\mathrm{HCO}^{+} 3-2$ had been observed as part of the survey for infall signatures by Gregersen et al. (2000). We used an SIS receiver with an acousto-optic spectrometer (AOS) with a $50 \mathrm{MHz}$ bandwidth and 1024 channels. The frequency resolution was about $2-2.5$ channels, i.e., $\sim 0.13 \mathrm{~km} \mathrm{~s}^{-1}$ at $220 \mathrm{GHz}$. The pointing uncertainty was approximately $4^{\prime \prime}$ on average. Table 1 also summarizes observational details of these data. 
TABLE 2

OVRO ObSERVATIONAL SUMmary

\begin{tabular}{|c|c|c|c|c|c|c|c|}
\hline Tracer & Transition & $\begin{array}{l}\text { Bandwidth } \\
\text { (MHz) }\end{array}$ & $\begin{array}{l}\text { Channel Spacing } \\
\qquad\left(\mathrm{km} \mathrm{s}^{-1}\right)\end{array}$ & $\begin{array}{l}\text { Gaussian Taper FWHM } \\
(\operatorname{arcsec} \times \operatorname{arcsec})\end{array}$ & $\begin{array}{l}\text { Synthesized Beam FWHM } \\
(\operatorname{arcsec} \times \operatorname{arcsec})\end{array}$ & $\begin{array}{c}\text { Synthesized Beam P.A. } \\
\text { (deg) }\end{array}$ & $\begin{array}{c}1 \sigma \mathrm{rms}^{\mathrm{a}} \\
\left(\mathrm{Jy} \mathrm{beam}^{-1}\right)\end{array}$ \\
\hline $\mathrm{H}_{2} \mathrm{CO} \ldots \ldots \ldots \ldots$ & $3_{12}-2_{11}$ & 7.00 & 0.17 & $1.60 \times 1.60$ & $2.5 \times 2.3$ & 304 & 0.1 \\
\hline $\mathrm{N}_{2} \mathrm{H}^{+} \ldots \ldots \ldots \ldots$ & $1-0$ & 7.50 & 0.20 & $4.0 \times 4.0$ & $6.5 \times 5.2$ & 334 & 0.07 \\
\hline
\end{tabular}

a $1 \sigma \mathrm{rms}$ computed from signal-free regions of the deconvolved maps.

\subsection{The Caltech Owens Valley Radio Observatory (OVRO) Millimeter Array Observations}

L1251B was observed in several transitions and continuum emission in the 1 or $3 \mathrm{~mm}$ bands of the OVRO Millimeter Array near Big Pine, CA, over several observing seasons. The continuum data have been presented in Paper I. Table 2 summarizes the line data, i.e., the frequencies observed and the bandwidths used, as well as the velocity channel spacings, the synthesized beam FWHMs, and the $1 \sigma$ rms values achieved.

In fall 1997 and spring 1998, $\mathrm{H}_{2} \mathrm{CO} J_{K_{-1} K_{+1}}=3_{12}-2_{11}$ at 225.6977750 GHz (Pickett et al. 1998) was observed over two $L$-configuration tracks and one $H$-configuration track for spatial frequency coverages of $\sim 11-156 \mathrm{k} \lambda$. These data were retrieved from the OVRO archive. In fall 1998 and spring 1999, $\mathrm{HCO}^{+}$ $1-0$ at $89.1885230 \mathrm{GHz}$ (Pickett et al. 1998) was observed over three $L$-configuration tracks, for spatial frequency coverages of 4-35 k $\lambda$. These data were also retrieved from the OVRO archive. In spring 2001 and fall $2001, \mathrm{~N}_{2} \mathrm{H}^{+} 1-0$ at $93.1762650 \mathrm{GHz}^{6}$ (Caselli et al. 1995) was observed over five $L$-configuration and one $H$-configuration tracks, for spatial frequency coverage of 4-64 k $\lambda$. These data were obtained specifically for this project. Refer to Paper I for details about observations and data processing.

\subsection{The SMA Observations}

L1251B was observed on 2005 September 25 from the summit of Mauna Kea, HI, with the SMA in its most compact configuration. The $230 \mathrm{GHz}$ SMA receiver was tuned to observe $\mathrm{CO}$ $2-1,{ }^{13} \mathrm{CO} 2-1, \mathrm{C}^{18} \mathrm{O} 2-1, \mathrm{~N}_{2} \mathrm{D}^{+} 3-2$, SO $5_{6}-45$, and $\mathrm{H}_{2}{ }^{13} \mathrm{CO}$ $3_{12}-2_{11}$ in separate correlator windows of various bandwidths and channel spacings. Table 3 lists the respective frequencies, sidebands of observation, correlator windows, bandwidths, and channel spacings for these lines. Out of the 24 windows in each sideband, the remaining correlator windows were used to ob-

\footnotetext{
$693.1762650 \mathrm{GHz}$ is the frequency of the $\mathrm{N}_{2} \mathrm{H}^{+} 101-012$ transition found by Caselli et al. (1995). The other six hyperfine components to the $1-0$ transition, at frequencies $<6 \mathrm{MHz}$ from that of 101-012, were also observed.
}

serve continuum emission over effectively $\sim 2 \mathrm{GHz}$ in each sideband. The continuum data have been presented in Paper I. Refer to Paper I for general information on observations and data processing.

We used the MIRIAD software package for imaging and deconvolution. For the $\mathrm{CO}$ and ${ }^{13} \mathrm{CO}$ data, natural weighting was used without any tapering to make the respective data cubes. The $\mathrm{C}^{18} \mathrm{O}$ and $\mathrm{N}_{2} \mathrm{D}^{+}$data, however, were tapered with a circular Gaussian of $4^{\prime \prime}$ FWHM during inversion to increase the beam size up to $\sim 5^{\prime \prime}$ FWHM for improved brightness sensitivity. Furthermore, only 20 velocity channels around the central velocity were used for the integrated intensity calculation to bring out low-brightness features. Finally, the $\mathrm{SO}$ and $\mathrm{H}_{2}^{13} \mathrm{CO}$ data were tapered with Gaussians of $6^{\prime \prime}$ FWHM, also to improve brightness sensitivity, but no detections of either line were obtained. To calculate the integrated intensities, all channels were used with a $2 \sigma$ rms clip.

\subsection{Data from Other Observations}

J. Williams (2005, private communication) provided us with unpublished CS 3-2 and 5-4 data that were obtained with the NRAO $12 \mathrm{~m}^{\text {telescope }}{ }^{7}$ on Kitt Peak, Arizona, in 1996 May (CS 5-4) and 1997 October (CS 3-2). The back end used was the Millimeter AutoCorrelator (MAC) with a channel spacing of $\sim 48.8 \mathrm{kHz}\left(\sim 0.1 \mathrm{~km} \mathrm{~s}^{-1}\right.$ for CS $2-1$ and $\sim 0.06 \mathrm{~km} \mathrm{~s}^{-1}$ for CS 5-4). Typical system temperatures were $250-300 \mathrm{~K}$ (CS 3-2) and $450-600 \mathrm{~K}$ (CS 5-4). Details of these line observations are also summarized in Table 1 .

\section{RESULTS}

In this study, we use coordinates in the J2000.0 epoch, so the $(\alpha, \delta)$ coordinates of IRAS $22376+7455$, the IRAS point source associated with L1251B, are $\left(22^{\mathrm{h}} 38^{\mathrm{m}} 47.16^{\mathrm{s}},+75^{\circ} 11^{\prime} 28.71^{\prime \prime}\right)$.

\footnotetext{
7 The Kitt Peak 12 m telescope was operated by NRAO, a facility of the National Science Foundation, operated under cooperative agreement by Associated Universities, Inc. It is currently operated by the Arizona Radio Observatory (ARO), Steward Observatory, University of Arizona, with partial funding from the Research Corporation.
}

TABLE 3

SMA Line ObSERVATions

\begin{tabular}{|c|c|c|c|c|c|c|c|}
\hline Line & $\begin{array}{c}\text { Frequency }^{\mathrm{a}} \\
\quad(\mathrm{GHz})\end{array}$ & S Band & Window & Channels & $\begin{array}{c}\Delta V \\
\left(\mathrm{~km} \mathrm{~s}^{-1}\right)\end{array}$ & $\begin{array}{c}\text { Beam FWHM } \\
(\operatorname{arcsec} \times \operatorname{arcsec})\end{array}$ & $\begin{array}{l}\text { P.A. } \\
\text { (deg) }\end{array}$ \\
\hline 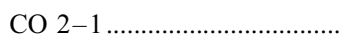 & 230.5380000 & USB & 14 & 128 & 1.06 & $4.1 \times 3.1$ & -26.2 \\
\hline${ }^{13} \mathrm{CO} 2-1$ & 220.3986765 & LSB & 13 & 512 & 0.26 & $4.3 \times 3.4$ & -23.5 \\
\hline 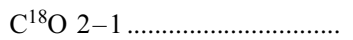 & 219.5603568 & LSB & 23 & 512 & 0.26 & $5.3 \times 5.0^{b}$ & 0.50 \\
\hline $\mathrm{N}_{2} \mathrm{D}^{+} 3-2 \ldots \ldots \ldots \ldots$ & 231.3216650 & USB & 23 & 512 & 0.26 & $5.1 \times 5.0^{b}$ & 10.4 \\
\hline 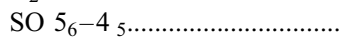 & 219.9494420 & LSB & 18 & 256 & 0.53 & $8.0 \times 6.3^{b, c}$ & 68.3 \\
\hline $\mathrm{H}_{2}^{13} \mathrm{CO} 3_{12}-2_{11} \ldots \ldots \ldots \ldots \ldots \ldots \ldots$ & 219.9085250 & LSB & 19 & 256 & 0.53 & $8.0 \times 6.3^{\mathrm{b}, \mathrm{c}}$ & 68.3 \\
\hline
\end{tabular}

a All line frequencies listed were obtained from Pickett et al. (1998)

b Beam attained after tapering with a $4^{\prime \prime} \times 4^{\prime \prime}$ FWHM Gaussian.

c Beam attained after tapering with a $6^{\prime \prime} \times 6^{\prime \prime}$ FWHM Gaussian. 


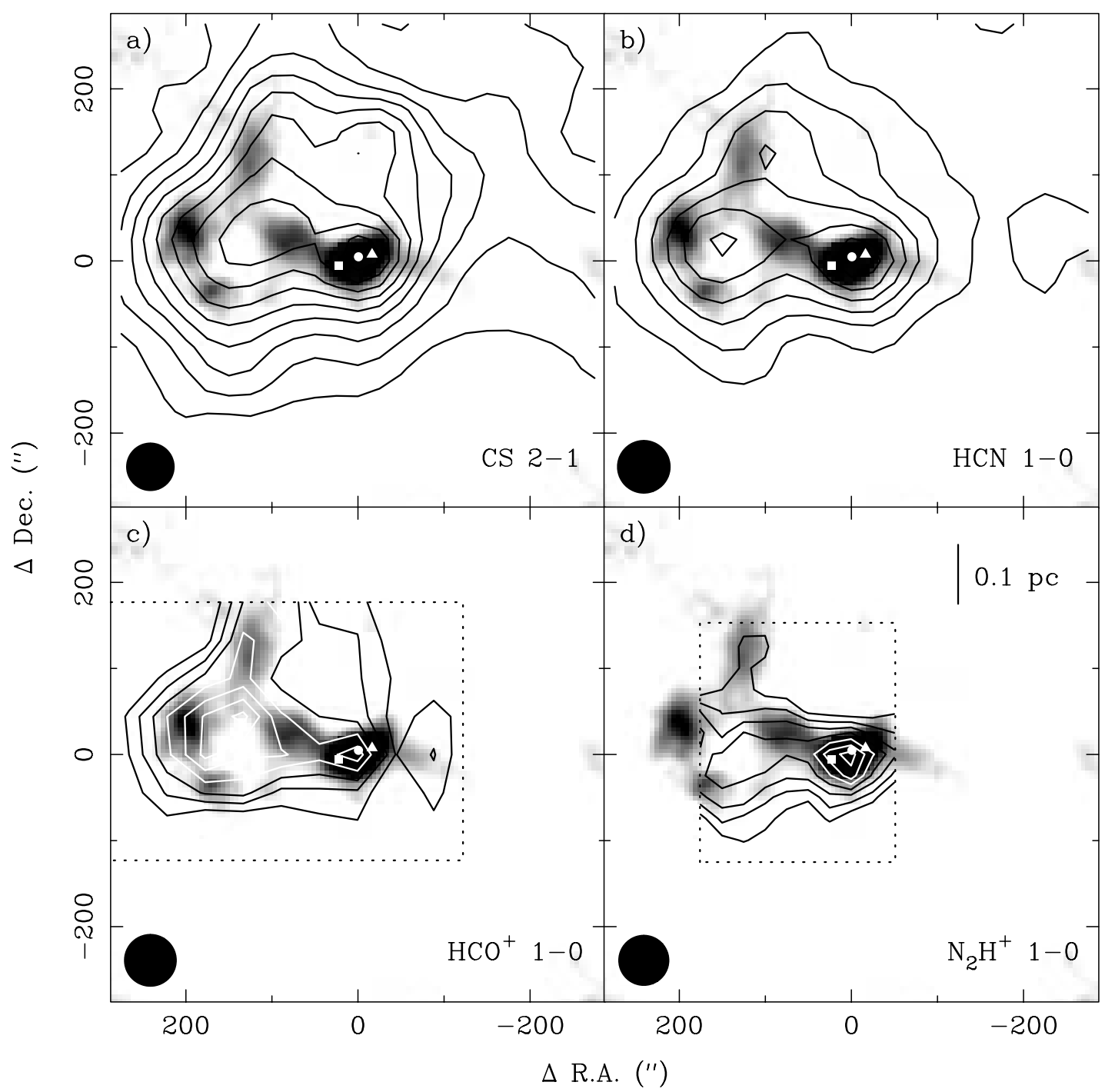

FIG. 1.- Integrated intensity maps (contours) of $\mathrm{CS} 2-1, \mathrm{HCN} 1-0, \mathrm{HCO}^{+} 1-0$, and $\mathrm{N}_{2} \mathrm{H}^{+} 1-0$ on top of the $850 \mu$ m emission map $($ gray scale). The $(0,0)$ position of this map represents the coordinates of IRAS $22376+7455$. In each panel, the small white circle, square, and triangle denote the positions of IRS1, IRS2, and IRS4, respectively. The gray scale used ranges from 0.084 to $0.420 \mathrm{Jy} \mathrm{beam}^{-1}$, roughly $2-10 \sigma$, where $1 \sigma \approx 0.042 \mathrm{Jy} \mathrm{beam}^{-1}$. In each panel, the large black circle at the lower left shows the beam FWHM. The dotted rectangles show the extent of the map in the respective line. (For CS $2-1$ and HCN 1-0, the map was larger than the area shown.) (a) Integrated intensity map of CS $2-1$. The velocity range used is -6.5 to $-0.5 \mathrm{~km} \mathrm{~s}^{-1}$. The contours begin at $10 \sigma$ and increase by $6 \sigma$, where $1 \sigma=0.06 \mathrm{~K} \mathrm{~km} \mathrm{~s}{ }^{-1}$. (b) Integrated intensity map of $\mathrm{HCN} 1-0$. The velocity range used is -13.5 to $4 \mathrm{~km} \mathrm{~s}^{-1}$. The contours begin at $10 \sigma$ and increase in steps of $5 \sigma$, where $1 \sigma=0.106 \mathrm{~K} \mathrm{~km} \mathrm{~s}^{-1}$. (c) Integrated intensity map of $\mathrm{HCO}^{+} 1-0$. The velocity range used is -6 to $-2 \mathrm{~km} \mathrm{~s}^{-1}$. The contours begin at $6 \sigma$ and increase in steps of $1.5 \sigma$, where $1 \sigma=0.42 \mathrm{~K} \mathrm{~km} \mathrm{~s} \mathrm{~s}^{-1}$. $(d)$ Integrated intensity map of $\mathrm{N}_{2} \mathrm{H}^{+} 1-0$. The velocity range used is -13 to $4 \mathrm{~km} \mathrm{~s}^{-1}$. The contours begin at $15 \sigma$ and increase in steps of $5 \sigma$, where $1 \sigma=0.166 \mathrm{~K} \mathrm{~km} \mathrm{~s}$.

This position defines the reference center for all figures that use offsets in their axes. For example, IRS1 is located at $\left(0^{\prime \prime},+5^{\prime \prime}\right)$.

\subsection{Observations with Single-Dish Telescopes}

Figure 1 presents the integrated intensity maps of CS 2-1, $\mathrm{HCN} 1-0, \mathrm{HCO}^{+} 1-0$, and $\mathrm{N}_{2} \mathrm{H}^{+} 1-0$ obtained at FCRAO. In general, these lines trace moderately dense $\left(n \leq 3 \times 10^{5} \mathrm{~cm}^{-3}\right)$ gas, i.e., they have low excitation requirements compared to other lines in this study. In each panel, line emission is shown overlaid onto a map of $850 \mu$ m continuum emission (see Paper I) and the locations of the YSOs IRS1, IRS2, and IRS4 are denoted by a circle, square, and triangle, respectively. Notably, the line maps of Figure 1 differ from the (low-resolution) map of integrated CS 1-0 intensity by Morata et al. (1997), which instead shows a maximum of emission $\sim 200^{\prime \prime}$ north of L1251B and no obvious structure around L1251B itself. These line maps are similar to that of $\mathrm{NH}_{3}$ integrated intensity by Tóth \& Walmsley (1996), however. Figure 1 shows that L1251B is coincident with line emission maxima in all four tracers, suggesting that it is associated with moderately dense gas. Significant line emission is also seen throughout the region beyond L1251B, however. For example, CS 2-1 shows a third maximum $\sim 130^{\prime \prime}$ north of L1251B, but no other tracer in Figure 1 shows a maximum toward that general location. In addition, other maxima are seen $\sim 100^{\prime \prime}-160^{\prime \prime}$ to the east of L1251B, associated with the east core identified from $850 \mu \mathrm{m}$ emission in Paper I. These eastern maxima are not coincident with each other, however. Also, a thin ridge of emission extends northward from the east core in all maps. The brightnesses of the maxima are similar for CS $2-1, \mathrm{HCN} 1-0$, and $\mathrm{H}^{13} \mathrm{CO}^{+} 1-0$ (not shown) in the east core and L1251B. The east core, however, is slightly brighter in $\mathrm{HCO}^{+} 1-0$ but much weaker in $\mathrm{N}_{2} \mathrm{H}^{+} 1-0$ than L1251B, although it was not mapped as extensively in the latter line.

Figure 2 presents the integrated intensity maps of CS $3-2$ and $5-4, \mathrm{HCO}^{+} 3-2$, and $\mathrm{DCO}^{+} 3-2$ obtained with the NRAO $12 \mathrm{~m}$ telescope or the CSO. In general, these lines trace gas of higher 


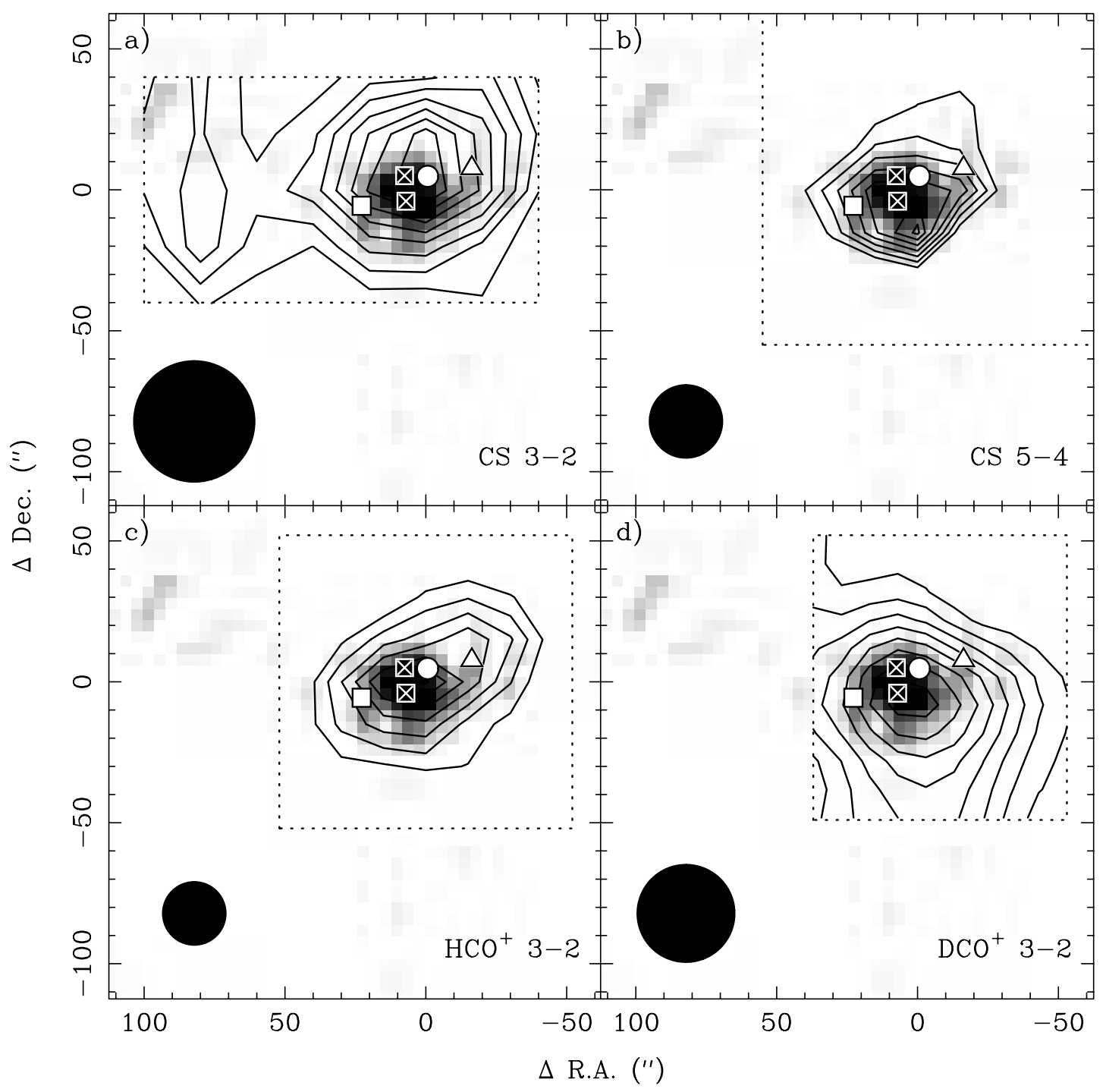

Fig. 2.- Integrated intensity maps (contours) of CS 3-2, 5-4, $\mathrm{HCO}^{+} 3-2$, and $\mathrm{DCO}^{+} 3-2$ on top of the $1.3 \mathrm{~mm}$ dust continuum emission map (gray scale). The gray scale ranges from 0.025 to $0.125 \mathrm{Jy}_{\text {beam }}^{-1}$ or about $2-10 \sigma$, where $1 \sigma=0.0125 \mathrm{Jy}_{\text {beam }}^{-1}$. In each panel, the dotted rectangle denotes the extent of the map in the respective line. Symbols are defined as in Fig. 1, but here the boxed cross symbols indicate positions of peak intensity of prestellar condensations detected in SMA $1.3 \mathrm{~mm}$ observations (see Fig. 8 of Paper I). (a) Integrated intensity map of CS 3-2. The velocity range used is -6.5 to $-0.5 \mathrm{~km} \mathrm{~s}^{-1}$. The contours begin at $15 \sigma$ and increase in steps of $5 \sigma$, where $1 \sigma=0.066 \mathrm{~K} \mathrm{~km} \mathrm{~s}^{-1}$. (b) Integrated intensity map of CS $5-4$. The velocity range used is -6.5 to $-0.5 \mathrm{~km} \mathrm{~s}^{-1}$. The contours begin at $10 \sigma$ and increase in steps of $4 \sigma$, where $1 \sigma=0.112 \mathrm{~K} \mathrm{~km} \mathrm{~s}^{-1}$. (c) Integrated intensity map of $\mathrm{HCO}^{+} 3-2$. The velocity range used is -8 to $0 \mathrm{~km} \mathrm{~s}^{-1}$. The contours begin at $3 \sigma$ and increase in steps of $3 \sigma$, where $1 \sigma=0.545 \mathrm{~K} \mathrm{~km} \mathrm{~s}^{-1}$. (d) Integrated intensity map of $\mathrm{DCO}^{+} 3-2$. The velocity range used is -5 to $-2.5 \mathrm{~km} \mathrm{~s}^{-1}$. The contours begin at $3 \sigma$ and increase in steps of $1 \sigma$, where $1 \sigma=0.097 \mathrm{~K} \mathrm{~km} \mathrm{~s}^{-1}$.

density $\left(n \geq 1 \times 10^{6} \mathrm{~cm}^{-3}\right)$ than those shown in Figure 1, i.e., they have relatively high excitation requirements. The maps of integrated intensity in Figure 2 are overlaid onto maps of $1.3 \mathrm{~mm}$ continuum emission, which traces the column density very well. (As described in Paper I, a shift in the location of the continuum emission maximum associated with L1251B to the south is seen with increasing wavelength.) The positions of IRS1, IRS2, and IRS4 are shown as in Figure 1, but here square-X symbols denote the locations the starless objects from Paper I, SMA-N, and SMA-S. Figure 2 shows that L1251B also contains maxima of higher excitation lines, suggesting even higher densities than those traced by the lines shown in Figure 1. (These maps, however, do not extend as widely as those in Fig. 1, so the larger scale distribution of these lines, e.g., toward the east core, is not known.) As in the single-dish continuum data, however, Figure 2 also shows that the integrated intensity maxima of dense gas tracers are located consistently off-center from IRS1. For example, the max- imum of CS $5-4$ is located $\sim 15^{\prime \prime}$ (greater than a half beam size) south of IRS1. These shifts seen in continuum and molecular emission maps suggest that within L1251B the maximum column density, and probably the density, resides between IRS1 and IRS2.

Figure 3 shows line profiles at the center position of L1251B of all eight lines from Figures 1 and 2 , plus $\mathrm{H}^{13} \mathrm{CO}^{+} 1-0$. The line widths of $\mathrm{DCO}^{+} 3-2, \mathrm{H}^{13} \mathrm{CO}^{+} 1-0$ and the isolated component of $\mathrm{N}_{2} \mathrm{H}^{+} 1-0$, which are obtained by Gaussian fits, are $\sim 1 \mathrm{~km} \mathrm{~s}^{-1}$. The line widths of $\mathrm{HCN} 1-0$ and $\mathrm{N}_{2} \mathrm{H}^{+} 1-0$, which are obtained by their hyperfine structure fits, are $\sim 1.4$ and $\sim 1.2 \mathrm{~km} \mathrm{~s}^{-1}$, respectively, probably affected by their optical depths. The width of CS $5-4$, however, is $\sim 2.6 \mathrm{~km} \mathrm{~s}^{-1}$. Line wings are seen in the spectra of $\mathrm{HCO}^{+} 3-2, \mathrm{CS} 5-4$, and $\mathrm{HCN}$ $1-0$. All profiles in Figure 3, except for $\mathrm{H}^{13} \mathrm{CO}^{+} 1-0, \mathrm{DCO}^{+}$ $3-2$, and the isolated component of $\mathrm{N}_{2} \mathrm{H}^{+} 1-0$ are asymmetric with blueshifted peak temperatures. The peak temperatures of the isolated component of $\mathrm{N}_{2} \mathrm{H}^{+} 1-0$ and $\mathrm{DCO}^{+} 3-2$ lines are 


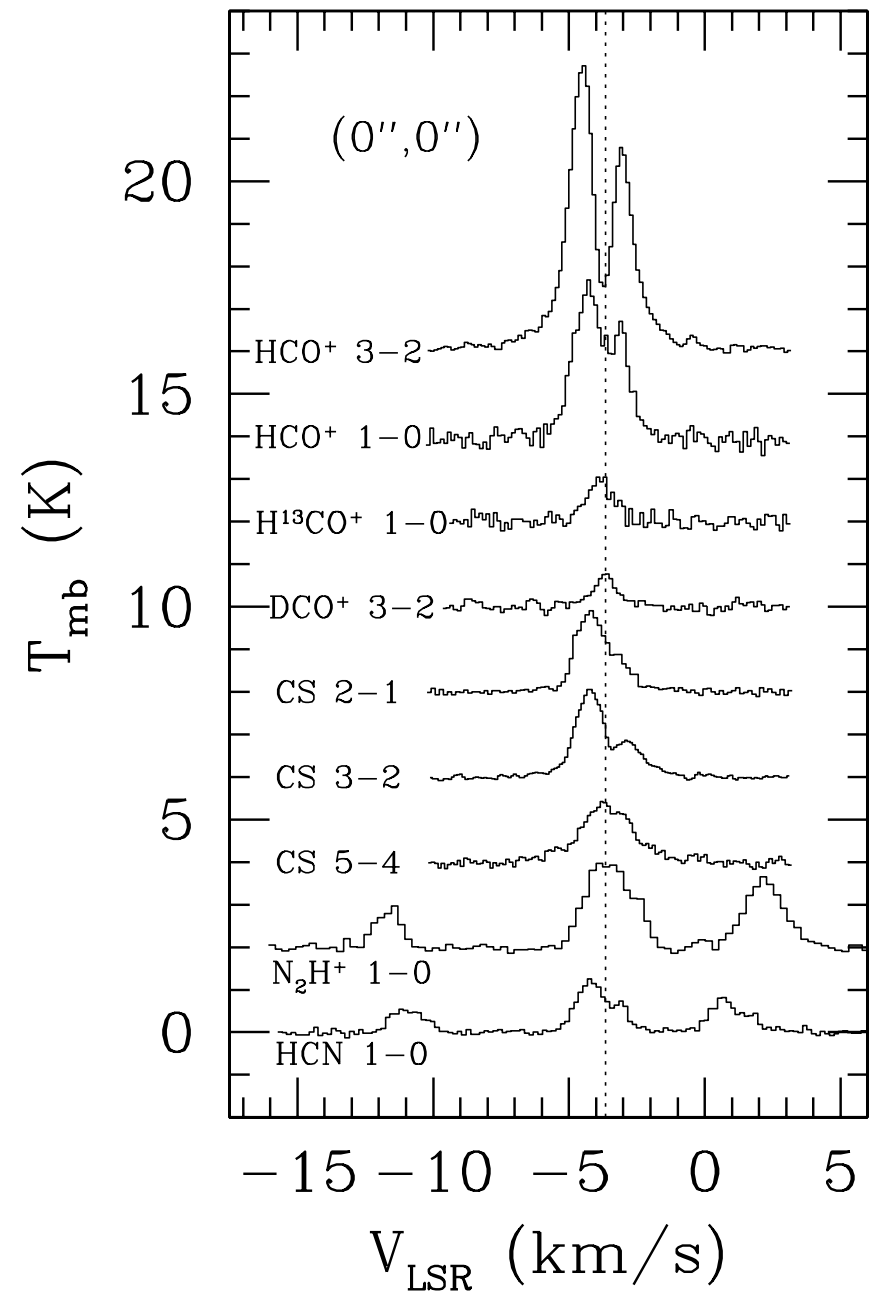

FIG. 3.- Spectra of all molecular transitions observed with single-dish telescopes ( Table 1) at the center position. To present line profiles better, the order of lines has been chosen arbitrarily, and spectra have been shifted up. The dotted vertical line indicates the centroid velocity of $\mathrm{L} 1251 \mathrm{~B},-3.65 \mathrm{~km} \mathrm{~s}^{-1}$.

located close to the central velocity of L1251B, i.e., where the deepest dips are seen in the other lines. We determined the central velocities of $\mathrm{L} 1251 \mathrm{~B}\left(-3.65 \mathrm{~km} \mathrm{~s}^{-1}\right)$ and the east core $\left(-4.13 \mathrm{~km} \mathrm{~s}^{-1}\right)$ by the Gaussian fitting of the isolated component of $\mathrm{N}_{2} \mathrm{H}^{+} 1-0$ and $\mathrm{H}^{13} \mathrm{CO}^{+} 1-0$, respectively. Figure 4 shows profiles of five lines toward the east core, where again asymmetric profiles with stronger blue peaks are seen in $\mathrm{HCO}^{+} 1-0, \mathrm{CS} 2-1$, and $\mathrm{HCN} 1-0$, but not $\mathrm{H}^{13} \mathrm{CO} 1-0$. Such profiles are not seen everywhere across the region, however, indicating a complex velocity distribution. For example, the CS 2-1 line profile (not shown) has reversed asymmetry toward the northern maximum of CS 2-1. HCN 1-0 (not shown) also has a reversed profile between the east core and L1251B.

Figure $5 a$ shows a map of red and blue components of $\mathrm{CO}$ 2-1 emission toward L1251B only, from data obtained with the CSO $10.4 \mathrm{~m}$ telescope. The velocity ranges for the red and blue components of the outflow have been determined very conservatively from blue- and red-free spectra (Fig. $5 b$ ).

The extended outflow from L $1251 \mathrm{~B}$, as seen by others, is plainly noticeable. The projected orientation of the $\mathrm{CO}$ outflow is northwest-southeast with blue or red components, respectively, in the northwest or southeast. The two components overlap significantly at $\mathrm{L} 1251 \mathrm{~B}$, however, and the origin of the outflow is not easily identifiable due to the low resolution of the map.

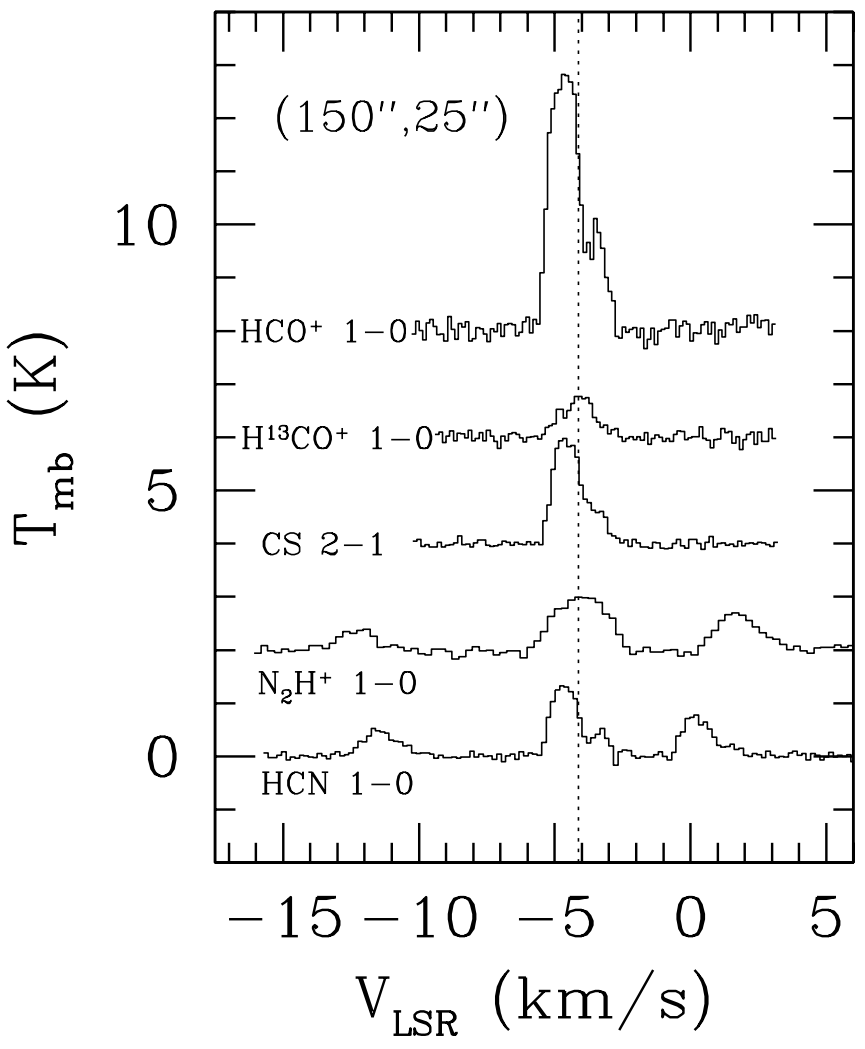

FIG. 4.- Spectra of some molecular transitions observed with single-dish telescopes toward the starless east core at $\left(150^{\prime \prime}, 25^{\prime \prime}\right)$. To present line profiles better, the order of lines has been chosen arbitrarily, and spectra have been shifted up. The dotted vertical line indicates the centroid velocity of the east core, $-4.13 \mathrm{~km} \mathrm{~s}^{-1}$, which is derived from the Gaussian line fitting of $\mathrm{H}^{13} \mathrm{CO}^{+} 1-0$.

Figure 6 shows $\mathrm{HCO}^{+} 3-2$ spectra from locations across L1251B. As seen in Figure 3, the central spectrum shows a very strong self-absorption dip and an asymmetric profile with a stronger blue peak. Figure 6 also reveals that the majority of lines off-center to L1251B have asymmetric profiles with stronger blue peaks, especially with increasing of the asymmetry with a stronger blue peak to the south. Not all the spectra are asymmetric with stronger blue peaks, however. To the north, the spectra are double peaked more symmetrically, and in the north reversed (red) asymmetry is seen. Note that the distribution of $\mathrm{HCO}^{+}$integrated intensities at L1251B (Fig. 2c) is elongated through the northwest-southeast direction. The outflow map (not shown) from the $\mathrm{HCO}^{+} 3-2$ wing components seen in Figure 3 shows the same trend as seen in the $\mathrm{CO} 2-1$ outflow map, i.e., a northwest-southeast extension and a significant positional overlap between the blue and red components.

\subsection{Interferometric Observations}

Figure 7 shows $\mathrm{HCO}^{+} 1-0, \mathrm{~N}_{2} \mathrm{H}^{+} 1-0$, and $\mathrm{H}_{2} \mathrm{CO} 33_{12}-2_{11}$ integrated intensities made with data from the OVRO MMA. Each map in Figure 7 is overlaid onto the Spitzer IRAC $4.5 \mu \mathrm{m}$ band image, and the positions of IRS1, etc., are denoted as in Figures 1 and 2 . In these cases, the maps only show smaller scale emission features since significant flux on larger scales was resolved out by the interferometer. Figure 7 shows that the distributions of compact line emission differ significantly on small scales. For example, Figure $7 a$ shows that the $\mathrm{HCO}^{+} 1-0$ emission is elongated linearly in the northwest-southeast direction from IRS1, similar to the direction of the outflow seen on larger scales (see Fig. 5). Figure $7 a$ also shows that the $\mathrm{N}_{2} \mathrm{H}^{+} 1-0$ emission is 

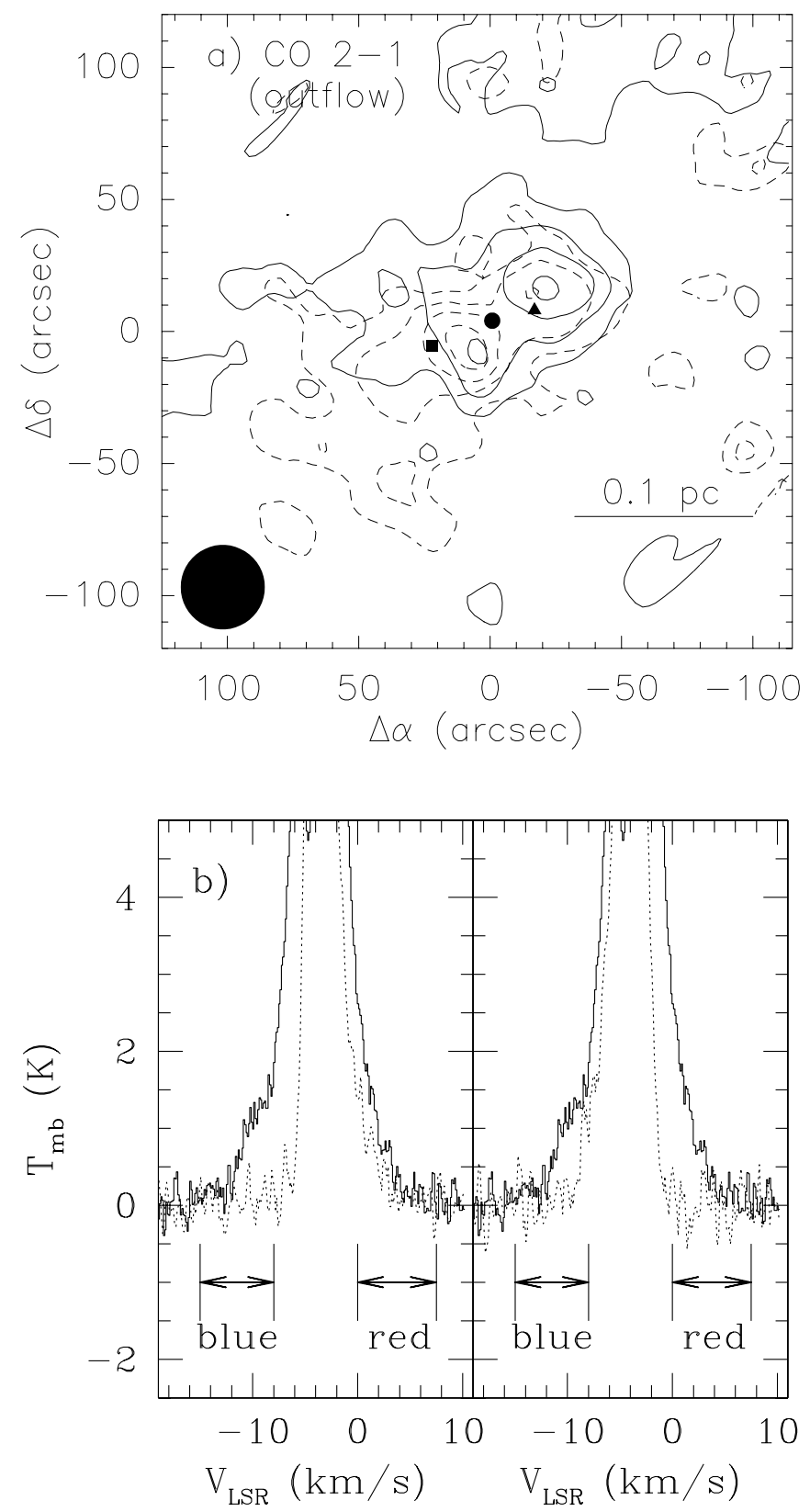

FIG. 5.- (a) Distribution of the outflow in L1251B in CO 2-1. Symbols are defined as in Fig. 1. The solid contours and dashed contours represent the blue and red components of the outflow, respectively. Contours are every $1 \mathrm{~K} \mathrm{~km} \mathrm{~s}^{-1}$ from $1 \mathrm{~K} \mathrm{~km} \mathrm{~s}^{-1}\left(1 \sigma=0.5 \mathrm{~K} \mathrm{~km} \mathrm{~s}^{-1}\right)$. The velocity range used for the blue and red components are -15 to $-8 \mathrm{~km} \mathrm{~s}^{-1}$ and 0 to $7.5 \mathrm{~km} \mathrm{~s}^{-1}$, respectively. (b) Spectrum at the center compared with blue- (left) and red-free (right) spectra. Arrows indicate the velocity ranges for the blue and red components.

located between IRS1 and IRS2 and is elongated in a projected direction perpendicular to that of the $\mathrm{HCO}^{+}$emission. In contrast, Figure $7 b$ shows compact $\mathrm{H}_{2} \mathrm{CO} 3_{12}-2_{11}$ emission in L1251B only at two locations, one centered at IRS1 and another $2^{\prime \prime}$ to the northeast of IRS4.

Figure 8 shows maps of $\mathrm{C}^{18} \mathrm{O} 2-1, \mathrm{~N}_{2} \mathrm{D}^{+} 3-2$, $\mathrm{CO} 2-1$, and ${ }^{13} \mathrm{CO} 2-1$ integrated intensities from $\mathrm{L} 1251 \mathrm{~B}$ with data from the SMA. Again, the observations were sensitive to only small-scale, compact features. The distributions of compact line emission again differ remarkably from each other and from those shown in Figure 7. Figure $8 a$ shows the $\mathrm{C}^{18} \mathrm{O} 2-1$ emission, along with $\mathrm{N}_{2} \mathrm{H}^{+} 1-0$ emission shown previously in Figure $7 a$. Compact ${ }^{18} \mathrm{O}$ emission is found along a filament containing IRS1 and IRS2, as well as SMA-N and SMA-S. Some $\mathrm{C}^{18} \mathrm{O}$ emission is also associated with IRS4. Note, however, that brighter $\mathrm{C}^{18} \mathrm{O}$ emission is slightly offset from all objects in L1251B. $\mathrm{N}_{2} \mathrm{H}^{+}$emission, however, is distributed in a direction perpendicular to the $\mathrm{C}^{18} \mathrm{O}$ filament, but its maxima are more coincident with SMA-N and SMA-S (but not completely; see $\S 4.4$ ). In contrast, Figure $8 b$ shows compact $\mathrm{N}_{2} \mathrm{D}^{+} 3-2$ emission, which is detected in a single location, south of IRS1 and slightly offset to the west of SMA-S. Figures $8 c$ and $8 d$ show the compact $\mathrm{CO} 2-1$ and ${ }^{13} \mathrm{CO} 2-1$ emission, which both show a lobelike structure extending southeast of IRS1. Bright CO emission is also seen to the northwest of IRS4, with a fainter extension coincident with IRS4. In contrast, bright ${ }^{13} \mathrm{CO}$ emission is coincident with IRS4 but is not seen to the northwest. Surprisingly, in these integrated intensity maps, neither CO nor ${ }^{13} \mathrm{CO}$ strong emission is seen associated with or coincident with IRS2, although its infrared nebulosity morphology is suggestive of an outflow cavity.

\section{ANALYSIS}

According to our observational results, complex dynamical and chemical processes are coupled in L1251B and the east core. To study each dynamical component, i.e., infall, rotation, and outflow, in detail, we describe in this section the analysis of line profiles using line radiative transfer calculations and the simulation of observed line profiles $(\S 4.1)$, centroid velocity maps and a position-velocity diagram ( $(4.2)$, and channel maps ( $(4.3)$, respectively. In addition, we calculate the chemical evolution coupled with the evolution of density and luminosity to compare the relative distribution of observed various molecular line emission with a chemical model (§ 4.4).

\subsection{Infall}

Toward L1251B, optically thick molecular transitions such as $\mathrm{HCO}^{+} 1-0$ and 3-2 show an asymmetrical profile with the brighter blue peak (see Fig. 3). In addition, optically thin molecular transitions such as $\mathrm{H}^{13} \mathrm{CO}^{+} 1-0$ and $\mathrm{DCO}^{+} 3-2$ lines are symmetrical, and peak at the velocities of the deepest dips of the other lines. Taken together, such profiles can be indicative of infall motions (see Zhou et al. 1993). In general, infalling gas will have motions both positive and negative along the line of sight, and if an excitation gradient also exists along the line of sight, redward self-absorption of gas emission can occur.

L1251B was modeled as an inside-out collapsing sphere (cf. Shu 1977) by Young et al. (2003), using continuum emission at 450 and $850 \mu \mathrm{m}$ to find density and temperature profiles of the core. The model parameters provided by Young et al., however, do not correspond to the best-fit model (C. Young 2005, private communication). Instead, the infall radius of the actual best-fit collapse model of L1251B is $5000 \mathrm{AU}$, equivalent to an infall timescale of $5 \times 10^{4} \mathrm{yr}$, rather than the $3000 \mathrm{AU}$ provided by Young et al. Other parameters of the best-fit model are the same, i.e., an effective sound speed of $0.46 \mathrm{~km} \mathrm{~s}^{-1}$ and a total luminosity of $10 L_{\odot}$. The model with a $5000 \mathrm{AU}$ infall radius provides much better spectral energy distribution (SED) fit. In the current best-fit model, the reduced $\chi^{2}$ for intensity profiles at 450 and $850 \mu \mathrm{m}$ and the SED are 17, 27, and 3, respectively (compare with $\chi^{2}$ in Tables 9 and 11 of Young et al.).

Figure 9 shows the density and velocity profiles of the best-fit inside-out collapse model. We have used the physical profiles to model $\mathrm{HCO}^{+} 3-2 / 1-0, \mathrm{H}^{13} \mathrm{CO}^{+} 1-0$, and $\mathrm{CS} 5-4$ lines with a Monte Carlo radiative transfer code (Choi et al. 1995). These lines trace the densest region, i.e., where the infall velocity is significant, among the lines observed with single-dish telescopes. To 


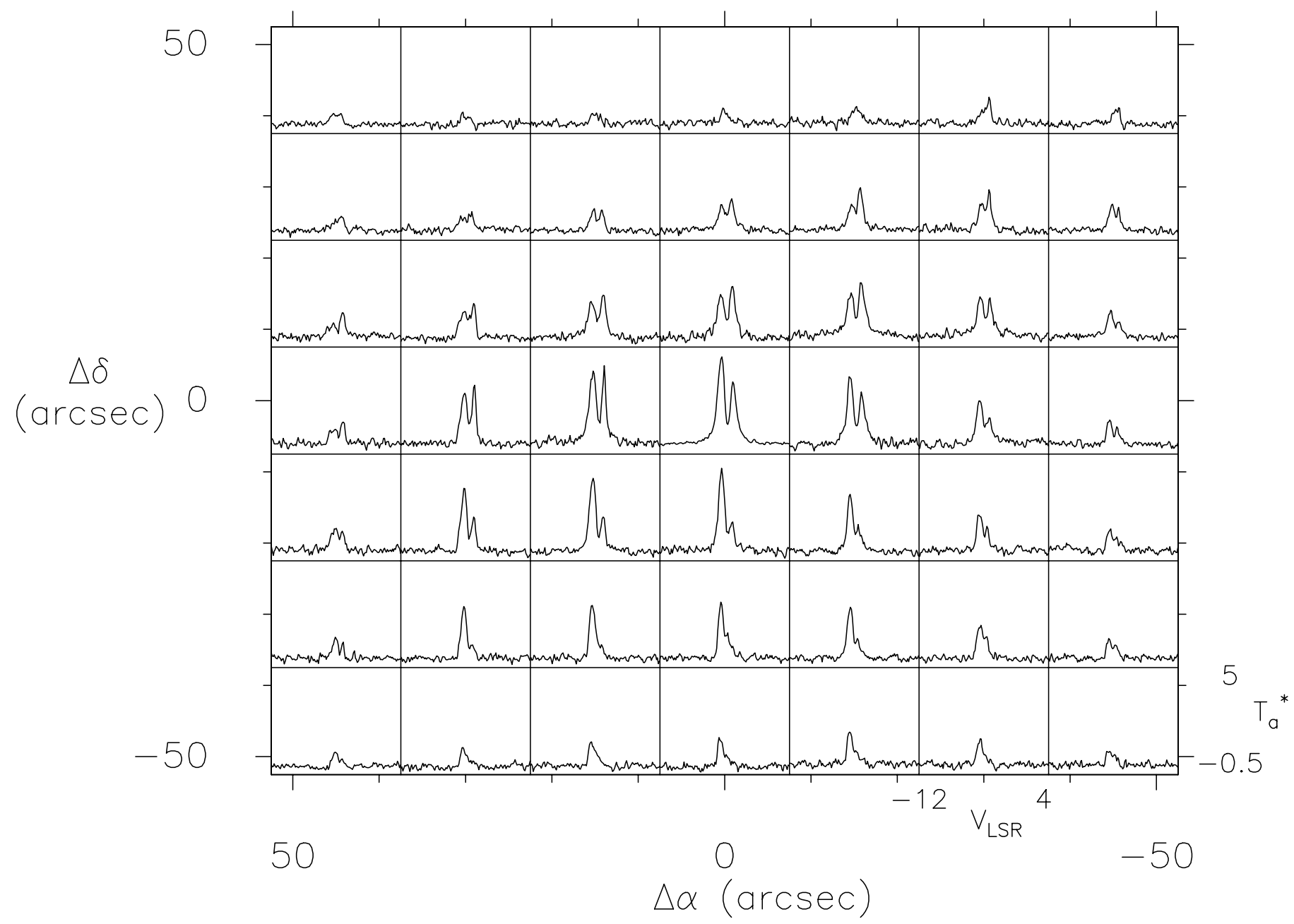

FIG. 6.- Spectra of $\mathrm{HCO}^{+} 3-2$ across L1251B. Panels are spaced by $15^{\prime \prime}$ in R.A. and decl. The horizontal axis is for $V_{\mathrm{LSR}}$, whose range is -12 to $4 \mathrm{~km} \mathrm{~s}{ }^{-1}$, and the vertical axis is in $T_{a}^{*}$, whose range is -0.5 to $5 \mathrm{~K}$.

minimize a contribution from rotational motions, line profiles observed only at the core center have been modeled. The kinetic temperature profile (Fig. 9a, dotted line) has been calculated with a gas energetics code (Young et al. 2004; Doty \& Neufeld 1997) from the dust temperature profile obtained from the dust continuum modeling. Abundance profiles of $\mathrm{HCO}^{+}$and $\mathrm{CS}$ were calculated with the chemo-dynamical model developed by Lee et al. (2004). (This chemical calculation and its results will be described in $\S$ 4.4.) Although an interferometric observation is necessary to study whether the high-velocity wings of CS 5-4 are affected by outflow, Figure 10 shows that the density and infall velocity structures from the best-fit model of dust continuum observations can reproduce the observed line profiles detected at the center of the core reasonably well, especially CS $5-4$. Note, however, that it was necessary to reduce the abundance profile of $\mathrm{HCO}^{+}$by a factor of 2 from that calculated by the chemical model, to fit the $\mathrm{HCO}^{+} 3-2 / 1-0$ line. The line profile of $\mathrm{H}^{13} \mathrm{CO}^{+}$ 1-0 was also fitted reasonably well with the $\mathrm{HCO}^{+}$abundance profile and the isotopic ratio of ${ }^{12} \mathrm{C} /{ }^{13} \mathrm{C}=77$ (Wilson \& Rood 1994). The dotted line in the figure presents the comparison case without infall. In this model, constant turbulent widths of 0.6 and $0.25 \mathrm{~km} \mathrm{~s}^{-1}$ (FWHM $=1$ and $0.4 \mathrm{~km} \mathrm{~s}^{-1}$, respectively) were assumed at radii respectively less than and greater than the infall radius. Having less turbulent motion in the outer stationary envelope than in the inner infalling envelope is necessary to fit the width of the self-absorption feature, especially that in $\mathrm{HCO}^{+} 3-2$.
The broad-line wings of $\mathrm{HCO}^{+} 3-2 / 1-0$ and $\mathrm{H}^{13} \mathrm{CO}^{+} 1-0$, particularly pronounced in the blueshifted emission, are probably caused by outflowing material, which is not considered in this model. The higher ratio between blue and red peaks of the observed line profiles than the modeled ones, which is commonly seen and never understood, might be affected by the rotational motion or the nonspherical geometry.

\subsection{Rotation}

Beyond the central position of L1251B, variations of the line profiles and position-velocity diagrams suggest rotational motions. For example, the $\mathrm{HCO}^{+} 3-2$ line profiles shown in Figure 6 at positions offset from $(0,0)$ show asymmetry reversals and increases that may be caused by rotational motions (e.g., see Walker et al. 1994; Zhou 1995; Ward-Thompson \& Buckley 2001). A simulation of the $\mathrm{HCO}^{+} 3-2$ spectra with a two-dimensional Monte Carlo molecular line radiative transfer code (Hogerheijde \& van der Tak 2000) for the collapse from a rotating dense cloud (Terebey et al. 1984) indeed produces the distribution of blue- and redasymmetric profiles observed in L1251B (Lee et al., in preparation). Note, however, that the wings of line profiles along the projected outflow direction may be still affected by outflow motions (see $\S 4.3$ below).

Previous studies (Goodman et al. 1993; Sato et al. 1994; Tóth \& Walmsley 1996; Anglada et al. 1997; Morata et al. 1997) determined that the overall velocity gradient in the L1251B 


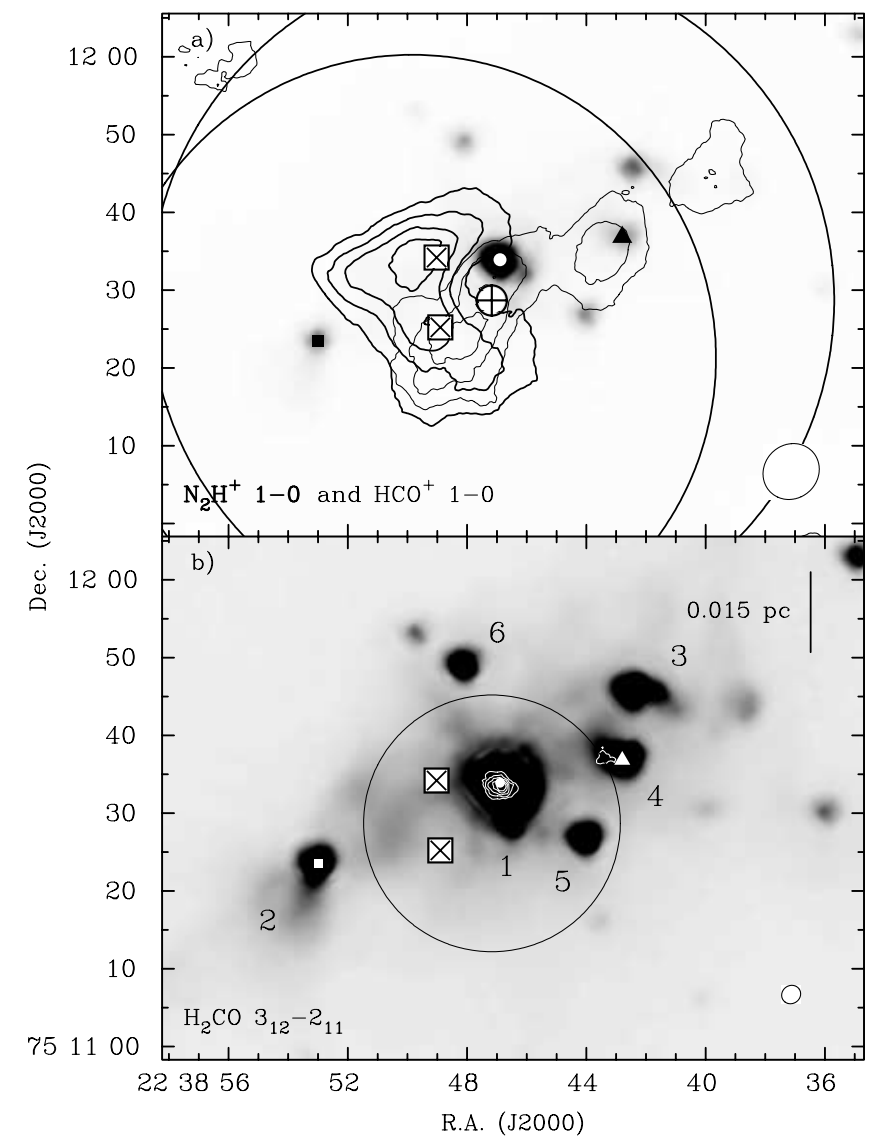

FIG. 7.- Integrated intensities of molecular transitions observed at OVRO, found by determining the "zeroth moment," i.e., summing over channels with emission $>|2| \sigma$. In all cases, no corrections for primary beam attenuation have been made. Each contour set begins at the respective $2 \sigma$ level and increases in steps of the respective $2 \sigma$. Integrated intensities are overlaid onto an image of the same region as seen in IRAC Band 2 (inverted gray scale). In each panel, the ellipse to the lower right denotes the size and P.A. of the synthesized beam FHWM. Symbols are defined as in Fig. 2, although the position of IRAS $22376+7455$ is shown as a circle-cross symbol. (a) $\mathrm{N}_{2} \mathrm{H}^{+} 1-0$ (thick contours) and $\mathrm{HCO}^{+} 1-0$ (thin contours), where $1 \sigma=1.5 \mathrm{~K} \mathrm{~km} \mathrm{~s}^{-1}$ for $\mathrm{N}_{2} \mathrm{H}^{+} 1-0$ and $1 \sigma=1.3 \mathrm{~K} \mathrm{~km} \mathrm{~s}^{-1}$ for $\mathrm{HCO}^{+} 1-0$. ( The beam FWHM shown is that of the $\mathrm{HCO}^{+} 1-0$ data.) The outer large circle shows the FWHM of the primary beam of the $\mathrm{HCO}^{+}$data, while the inner large circle shows the FWHM of the primary beam of the $\mathrm{N}_{2} \mathrm{H}^{+}$data. For the IRAC Band 2 data, the gray scale range is $-0.5-100 \mathrm{MJy} \mathrm{sr}^{-1}$. (b) Integrated intensities of $\mathrm{H}_{2} \mathrm{CO}_{3}{ }_{12}-2_{11}$ (contours), where $1 \sigma=0.54 \mathrm{~K} \mathrm{~km} \mathrm{~s}^{-1}$. The large circle shows the FWHM of the primary beam of $\mathrm{H}_{2} \mathrm{CO}$ data. For the IRAC Band 2 data, the gray scale range is $-0.5-10 \mathrm{MJy} \mathrm{sr}^{-1}$. The numbers shown identify individual infrared sources described in the text. (IRS1, IRS2, and IRS4 were classified as Class 0/I objects, and IRS3, IRS5, and IRS6 were classified as Class II sources in Paper I.)

neighborhood, i.e., that covered by the extended $850 \mu \mathrm{m}$ map in Figure 1, was $\sim 1-2 \mathrm{~km} \mathrm{~s}^{-1} \mathrm{pc}^{-1}$ in the northeast-southwest direction. Observations by Caselli et al. (2002), however, revealed a more complex velocity distribution in the neighborhood, with gradients of opposite direction. Namely, L1251B itself is associated with a southwest-northeast gradient, while the east core is associated with a northeast-southwest gradient. The average direction reported in their paper, however, is also northeast-southwest, suggesting that the previous studies with poorer resolutions averaged out the velocity gradient associated with L1251B, whose velocity gradient is only about half of that in the east core. To determine the velocity gradient associated with L1251B and the east core at higher resolution, we fitted the hyperfine structures of $\mathrm{HCN} 1-0$ and $\mathrm{N}_{2} \mathrm{H}^{+} 1-0$ and the Gaussian profile of $\mathrm{H}^{13} \mathrm{CO}^{+} 1-0$, and found the same result as shown in Figure 6 of Caselli et al. For illustration, Figure 11 shows the centroid velocity distribution obtained from $\mathrm{HCN}$ $1-0$, which shows different velocity gradients in L1251B and the east core. The derived velocity gradient from HCN $1-0$ in L1251B within $80^{\prime \prime}$ is $\sim 3 / \cos (i) \mathrm{km} \mathrm{s}^{-1} \mathrm{pc}^{-1}$ in the southwestnortheast direction but the velocity gradient in the east core is $\sim 6 / \cos (i) \mathrm{km} \mathrm{s}^{-1} \mathrm{pc}^{-1}$ in the opposite direction, i.e., consistent with the direction predicted from previous studies. Here, $i$ is the inclination of the rotational axis from the plane of sky.

We have analyzed the centroid velocity shift in L1251B with the $\mathrm{N}_{2} \mathrm{H}^{+} 1-0$ interferometer data (see Figs. $7 a$ and $8 a$ ). Figure 12 (top) shows a two-dimensional distribution of the mean centroid velocity with the integrated intensity contours of the isolated component of $\mathrm{N}_{2} \mathrm{H}^{+} 1-0$, which were calculated with the AIPS task, MOMNT. A clear shift of the centroid velocity is seen from the southwest to the northeast, along a direction perpendicular to the outflow direction. This southwest-northeast direction is opposite to the velocity gradient directions seen in previous studies with lower resolution, but consistent with the result of Caselli et al. (2002) and that seen in HCN 1-0 (Fig. 11) toward L1251B. A position-velocity diagram taken along a cut centered at $\left(9^{\prime \prime},-2^{\prime \prime}\right)$ and along P.A. $=33^{\circ}$ (similar to the direction of maximum elongation of the $\mathrm{N}_{2} \mathrm{H}^{+}$emission) is seen in Figure 12 (bottom). The rotational motion suggested by this gradient is very fast, i.e., $30 / \cos (i) \mathrm{km} \mathrm{s}^{-1} \mathrm{pc}^{-1}, \Omega \sim 10^{-12} / \cos (i) \mathrm{s}^{-1}$ within $30^{\prime \prime}$. This gradient is $\sim 1$ order of magnitude larger than what is seen on larger scales, such as in the HCN map (Fig. 11), suggesting an increased importance of rotation on small scales.

\subsection{Outflow}

In this section, we examine at high angular resolution the outflowing gas from the young stellar objects in L1251B, and provide an initial interpretation of the data. Although the evidence for outflows in this region is strong, the spatial distribution of this gas is very irregular, making it difficult to associate the observed outflow features with specific objects.

Figure 13 shows the $\mathrm{CO} 2-1$ integrated intensity across L1251B as observed from the SMA, divided into red and blue components (emission at line center is excluded). For reference, the CO components are overlaid onto the $1.3 \mathrm{~mm}$ continuum emission across L1251B, also observed from the SMA. Figures 14 and 15 show channel maps of $\mathrm{CO} 2-1$ respectively for red- and blueshifted emission. For reference, the $\mathrm{CO}$ emission in each channel is overlaid here onto the $\mathrm{N}_{2} \mathrm{H}^{+} 1-0$ integrated intensity as observed from the OVRO millimeter array. (Note that the velocity range definitions of red-and blueshifted emission are slightly different in these figures than those used for Figs. 5 and 13 because of the difference in their velocity resolutions.) In Figures 14 and 15, we also include more channel maps at $-1.2 \mathrm{~km} \mathrm{~s}^{-1}$, and -5.4 and $-6.5 \mathrm{~km} \mathrm{~s}^{-1}$, in the red- and blueshifted emission, respectively, to resolve different outflow components. For an alternative view of the outflows associated with L1251B, Figure 16 shows channel maps of $\mathrm{HCO}^{+} 1-0$ observed from OVRO. In the channel maps, especially for blueshifted emission (Figs. 15 and 16), velocities closer to the central velocity than defined conservatively in Figures 5 and 13 are covered. While the $\mathrm{HCO}^{+} 1-0$ emission around the central velocity peaks at SMA-S, which is the densest part in L 1251B, it has peaks at IRS1 and southeast of IRS4 with a weaker tail toward the northwest of IRS4, at -5.2 and $-5.6 \mathrm{~km} \mathrm{~s}^{-1}$. Therefore, $\mathrm{HCO}^{+} 1-0$ mainly traces the outflowing material even at the velocity up to $\sim-5 \mathrm{~km} \mathrm{~s}^{-1}$. In addition, the comparison between the model and observation of $\mathrm{HCO}^{+} 1-0$ in Figure $5 b$ suggests that the emission at $-5 \mathrm{~km} \mathrm{~s}^{-1}$ is affected only by the outflow. 


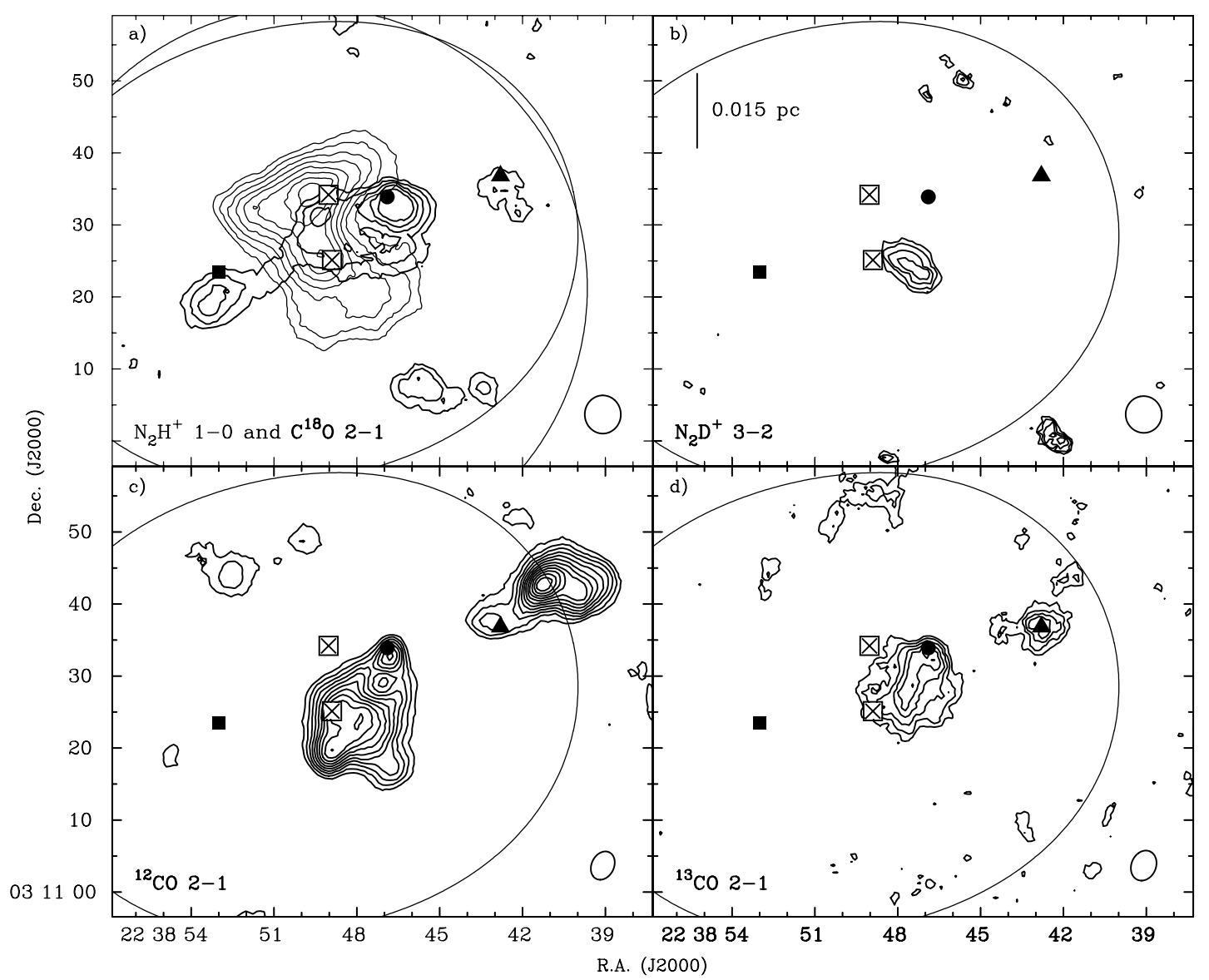

FIG. 8. - Integrated intensities of molecular transitions observed at SMA, found by determining the zeroth moment, i.e., summing over channels with emission $>|2| \sigma$. Each contour set begins at the respective $2 \sigma$ level and increases in steps of respective $1 \sigma$. In each panel, the ellipse to the lower right denotes the size and P.A. of the respective synthesized beam FWHM. In each panel, the large oval denotes the extent of the half-power sensitivity of the two-pointing mosaic, obtained from the continuum data obtained when the lines were observed. (The primary beam attenuation was corrected when these mosaics were made.) Various symbols are defined as in previous figures. (a) Integrated intensities of $\mathrm{C}^{18} \mathrm{O} 2-1$ (thick contours) where $1 \sigma \approx 0.5 \mathrm{~K} \mathrm{~km} \mathrm{~s}^{-1}$. For comparison, the integrated intensities of $\mathrm{N}_{2} \mathrm{H}^{+} 1-0$ from Fig. $7 a$ are also shown. (The outer large circle denotes the primary beam FWHM of the $\mathrm{N}_{2} \mathrm{H}^{+}$data.) (b) Integrated intensities of $\mathrm{N}_{2} \mathrm{D}^{+} 3-2$ where $1 \sigma \approx 0.5 \mathrm{~K} \mathrm{~km} \mathrm{~s}^{-1}$. (The contours just south of the half-power sensitivity oval are tracing amplified noise and not $\mathrm{N}_{2} \mathrm{D}^{+}$emission.) Integrated intensities of ${ }^{12} \mathrm{CO} 2-1$ where $1 \sigma \approx 7 \mathrm{~K} \mathrm{~km} \mathrm{~s}{ }^{-1}$. (d) Integrated intensities of ${ }^{13} \mathrm{CO} 2-1$ where $1 \sigma \approx 2 \mathrm{~K} \mathrm{~km} \mathrm{~s}^{-1}$.

As can be seen from Figure 13, significant outflow appears to be associated with IRS1. This emission, however, may also include a component from SMA-S. Bright emission is seen extending southeast of IRS1, with blueshifted emission to the east-southeast and redshifted emission to the south-southeast and some positional overlap of these components to the southeast. The overlap of the components could indicate a single outflow where the inclination of the axis from the plane of sky is not large and the blue and red components, respectively, trace the front and back components of an outflow cone. Note, however, that in the redshifted $-1.2 \mathrm{~km} \mathrm{~s}^{-1}$ channel of Figure 14 the feature divides into two components, one associated to the west and southwest of IRS1 and another to the southwest of SMA-S. Note also the pair of distinct components seen in Figure 15, associated with IRS1 in one blueshifted pair of channels at -5.4 and $-6.5 \mathrm{~km} \mathrm{~s}^{-1}$ and associated with SMA-S in another blueshifted pair of channels at -9.6 and $-11 \mathrm{~km} \mathrm{~s}^{-1}$. In the latter case, the emission may have been made compact and elongated in the northwest-southeast direction due to higher densities to its immediate northeast, as evident from the $\mathrm{N}_{2} \mathrm{H}^{+}$emission and the higher excitation line emission (see Fig. 2).

For IRS2, a single redshifted feature is seen extending to its southeast in Figure 13; this feature is also seen in the $\mathrm{CO}$ channel maps from 2 to $-1.2 \mathrm{~km} \mathrm{~s}^{-1}$ of Figure 14. No corresponding blueshifted feature is obviously associated with IRS2, however. It is possible that the blueshifted feature near IRS1 seen at -5.4 and $-6.5 \mathrm{~km} \mathrm{~s}^{-1}$ is associated with IRS2 instead. For example, $\mathrm{CO}$ may be severely depleted in the dense regions between IRS1 and IRS2, and the blueshifted outflow from IRS2 may be only visible in CO near IRS1 because of localized high CO abundance due to the evaporation of $\mathrm{CO}$ from nearby dust grains. Note, however, that this blueshifted emission lies in a similar northwest-southeast direction as redshifted emission from IRS1. Alternatively, the extended redshifted feature southeast of IRS2 may be related to IRS1 itself.

Northwest of IRS4, Figure 13 shows red- and blueshifted features with very significant positional coincidence. Again, these features could be due to a single outflow of low inclination. The channel maps of Figures 14 and 15 suggest a more complex interpretation, however, with weak red- and blueshifted emission seen both northwest and east of IRS4 at $-1.2,-5.4$, and $-6.5 \mathrm{~km} \mathrm{~s}^{-1}$. Figure 16 shows blueshifted emission from $\mathrm{HCO}^{+}$ $1-0$, also located to the east and the northwest of IRS4. Instead, there may be two outflows present, one centered at IRS4 and another to the northwest. The latter may originate from IRS1 and could be the counterpart to the redshifted emission seen southeast of IRS2. Note that not much material is seen between IRS4 and IRS1 in the continuum emission maps of Figures 2 and 9 or 

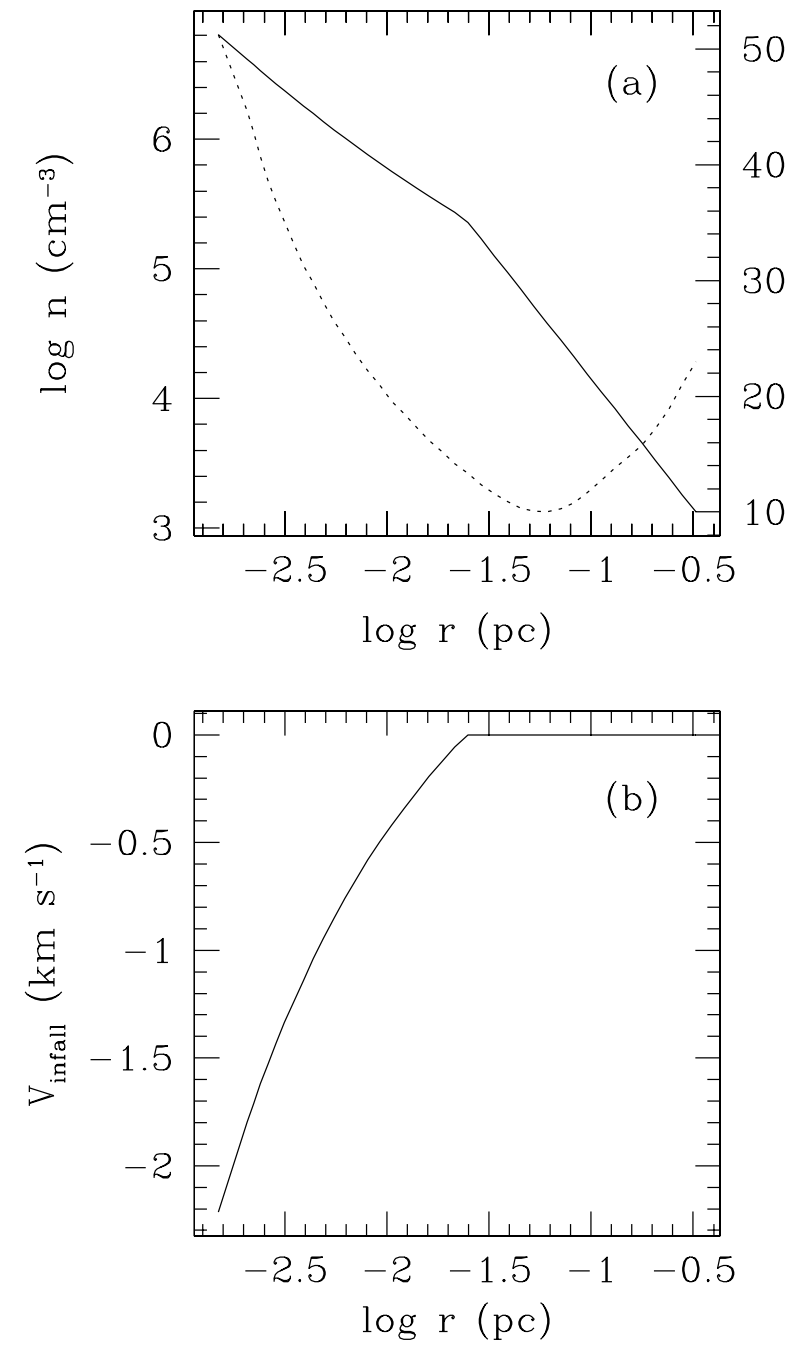

FIG. 9.- (a) Density and $(b)$ infall velocity profiles of the best-fit inside-out collapse model of L1251B for the dust continuum observations at 450 and $850 \mu \mathrm{m}$. The infall radius is $5000 \mathrm{AU}$, equivalent to an infall timescale of $5 \times 10^{4} \mathrm{yr}$. The kinetic temperature profile in $(a)$ has been calculated by balancing the heating and cooling of gas from the dust temperature profile obtained from the dust continuum modeling. (See Young et al. [2003] for details of the dust modeling process.) At small radii the kinetic temperature is well coupled with the dust temperature, but at large radii it is higher than the dust temperature, mainly due to the photoelectric heating.

the $\mathrm{CO} 2-1$ channel maps of Figure 13. Compact $\mathrm{HCO}^{+} 1-0$ emission is seen between IRS4 and IRS1, however, in Figure 16 from -6 to $-5 \mathrm{~km} \mathrm{~s}^{-1}$. The strongest $\mathrm{HCO}^{+}$emission is seen southeast of IRS4, and this may constitute a blueshifted outflow component from IRS4.

A summary of the possible outflows in the L1251B region is shown in Figure 17, with outflow components schematically plotted over the $1.3 \mathrm{~mm}$ continuum emission observed with the SMA. In this picture, IRS1, IRS2, IRS4, and even SMA-S all have outflows, some of which interact with each other. If SMA-S indeed has an outflow, it is not a starless condensation after all. Instead, it may harbor a very low luminosity protostellar object that was undetected by Spitzer. The existence of such objects has been surmised by others; e.g., Rebull et al. (2007) suggested that several objects unseen at $\lambda<70 \mu \mathrm{m}$ could be driving numerous outflows observed in HH 211. Future observations of L1251B, at even higher resolutions or using various lines that clearly trace gas shocked by outflow motions (e.g., mid-infrared lines of $\mathrm{H}_{2}$ or millimeter lines of $\mathrm{SiO}$ ), will be very helpful in disentangling
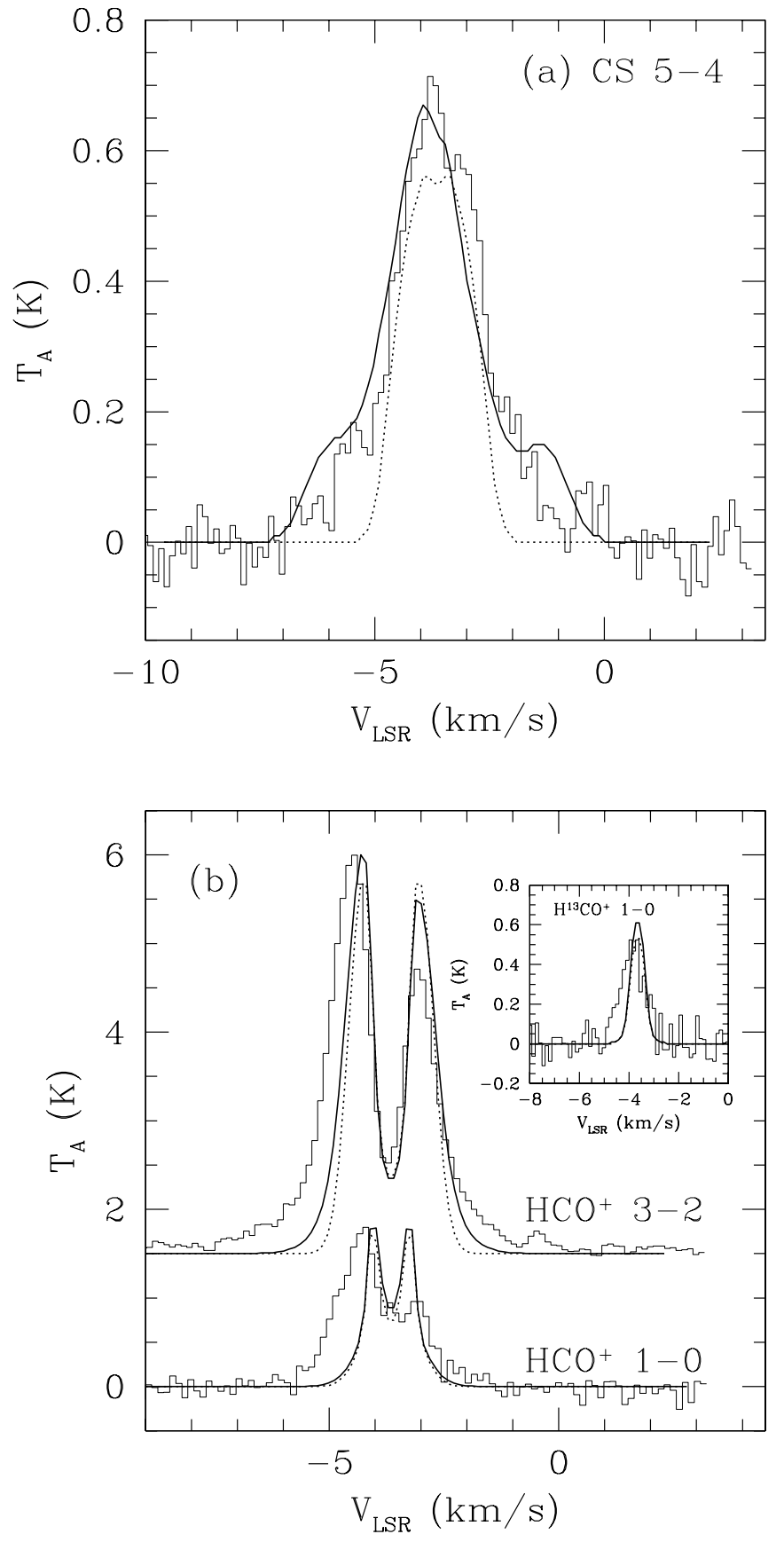

Fig. 10.-Comparisons between observations (histogram) and models with (solid line) and without (dotted line) the consideration of infall velocity profile in (a) CS 5-4 and $(b) \mathrm{HCO}^{+} 3-2$ and $\mathrm{H}^{13} \mathrm{CO}^{+} 1-0$. For the line radiative transfer calculations, physical conditions in Fig. 9 have been used, and the abundance profiles were adopted from Fig. 18. For the $\mathrm{H}^{13} \mathrm{CO}^{+}$abundance profile, the abundance ratio, $12 \mathrm{C} /{ }^{13} \mathrm{C}=77$ was assumed, and the $\mathrm{HCO}^{+}$abundance profile for $\mathrm{HCO}^{+} 3-2$ has been reduced by a factor of 2 from the profile seen in Fig. 18. The infall velocity profile is important to produce the broad wings of CS $5-4$, which traces very high densities. The line wings of $\mathrm{HCO}^{+} 3-2$ and $\mathrm{H}^{13} \mathrm{CO}^{+} 1-0$ may also be affected by outflow, which is not considered in this modeling.

the association of the outflows with specific sources and their effects on the evolution of the L1251B core.

\subsection{Chemistry}

Within L1251B, our high-resolution maps provide continued evidence for the expected chemical behavior of dense gas not associated with protostars. For example, the $\mathrm{HCO}^{+}$features shown in Figure $7 a$ likely traces outflowing dense gas, since $\mathrm{HCO}^{+}$can be 


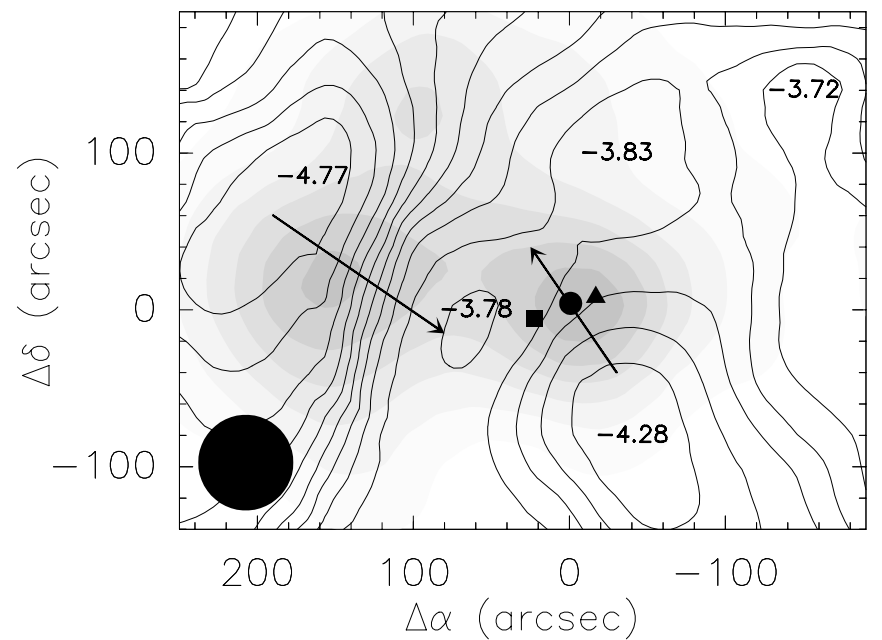

FIG. 11.-Centroid velocity distribution of $\mathrm{HCN} 1-0$ as observed from FCRAO overlaid onto its integrated intensity map. Centroid velocities were calculated by fitting the hyperfine structure of HCN $1-0$. The interval of contours is $0.1 \mathrm{~km} \mathrm{~s}^{-1}$ and numbers indicate local extrema of centroid velocity. The arrows indicate the directions of velocity gradients from blue to red. The uncertainty of fitting of the centroid velocity is less than $0.1 \mathrm{~km} \mathrm{~s}^{-1}$.

made abundant through shocks (see Rawlings et al. 2004). As with $\mathrm{HCO}^{+}$, the $\mathrm{CO},{ }^{13} \mathrm{CO}$, and diffuse $\mathrm{C}^{18} \mathrm{O}$ emission seen in Figures $8 a$, $8 c$, and $8 d$ may also be due to extensive depletion in the extended envelope and enhancements along the outflow axis due to liberation from grains. Emission from these lines indeed shares some common features, but note that no compact $\mathrm{CO},{ }^{13} \mathrm{CO}$, or $\mathrm{C}^{18} \mathrm{O}$ emission is seen between IRS1 and IRS4. A faint ridge of $\mathrm{HCO}^{+}$ $1-0$ emission is seen at that location at the level of $2 \sigma$, however.

In addition, the $\mathrm{N}_{2} \mathrm{H}^{+}$feature shown in Figure $7 a$ likely traces nonoutflowing gas, since $\mathrm{N}_{2} \mathrm{H}^{+}$can remain abundant in cold dense material and can be depleted significantly in outflows. $\mathrm{N}_{2} \mathrm{H}^{+}$is a chemical daughter of $\mathrm{N}_{2}$, which forms slowly. The interactions between $\mathrm{N}_{2} \mathrm{H}^{+}$and grains replenish $\mathrm{N}_{2}$ in the gas phase because $\mathrm{N}_{2} \mathrm{H}^{+}$recombines with electrons on the grain surfaces. The principal destroyers of $\mathrm{N}_{2} \mathrm{H}^{+}$in the gas phase are $\mathrm{CO}$ and electrons. $\mathrm{CO}$ can be significantly depleted in starless cores (Bergin \& Langer 1997) because the only source of heating is the interstellar radiation field and the inner temperatures of such cores can correspondingly be very low (e.g., $\sim 7 \mathrm{~K}$; see Evans et al. 2001). Also, the electron abundance can be very low $\left(\sim 10^{-9}\right.$; Williams et al. 1998) in dense gas that is highly extincted, like that in starless cores. The $\mathrm{N}_{2} \mathrm{H}^{+}$emission seen in Figures $7 a$ and $8 a$ is consistent with the $1.3 \mathrm{~mm}$ dust continuum emission mapped by the IRAM 30 m telescope (see Paper I), suggesting it is also tracing cold, dense gas.

$\mathrm{N}_{2} \mathrm{H}^{+}$(and $\mathrm{N}_{2} \mathrm{D}^{+}$) will also deplete at densities greater than $10^{6} \mathrm{~cm}^{-3}$ (Di Francesco et al. 2004; Pagani et al. 2005). Note that the two $\mathrm{N}_{2} \mathrm{H}^{+}$intensity maxima in Figures $7 a$ and $8 a$ are offset slightly to the east from SMA-N and SMA-S, which trace the densest parts of L1251B. ( The $\mathrm{C}^{18} \mathrm{O}$ emission observed with the SMA does not have maxima at SMA-N and SMA-S either.) In addition, an $\mathrm{N}_{2} \mathrm{D}^{+}$emission maximum is found between IRS1 and SMA-S. Finally, no such emission is associated with SMA-N. The offset of the $\mathrm{N}_{2} \mathrm{H}^{+}$maxima and deficiencies of $\mathrm{N}_{2} \mathrm{D}^{+}$from these objects are possibly caused by the depletion from the gas phase at densities greater than $10^{6} \mathrm{~cm}^{-3}$. The $\mathrm{N}_{2} \mathrm{D}^{+}$emission may be identifying a less dense location that has a high enough temperature to significantly populate $\mathrm{N}_{2} \mathrm{D}^{+}$at $J=3$. For example, the $J=3$ level of $\mathrm{N}_{2} \mathrm{D}^{+}$has its maximum population in thermal equilibrium at $\sim 20 \mathrm{~K}$, and this may be caused by heating by IRS1.
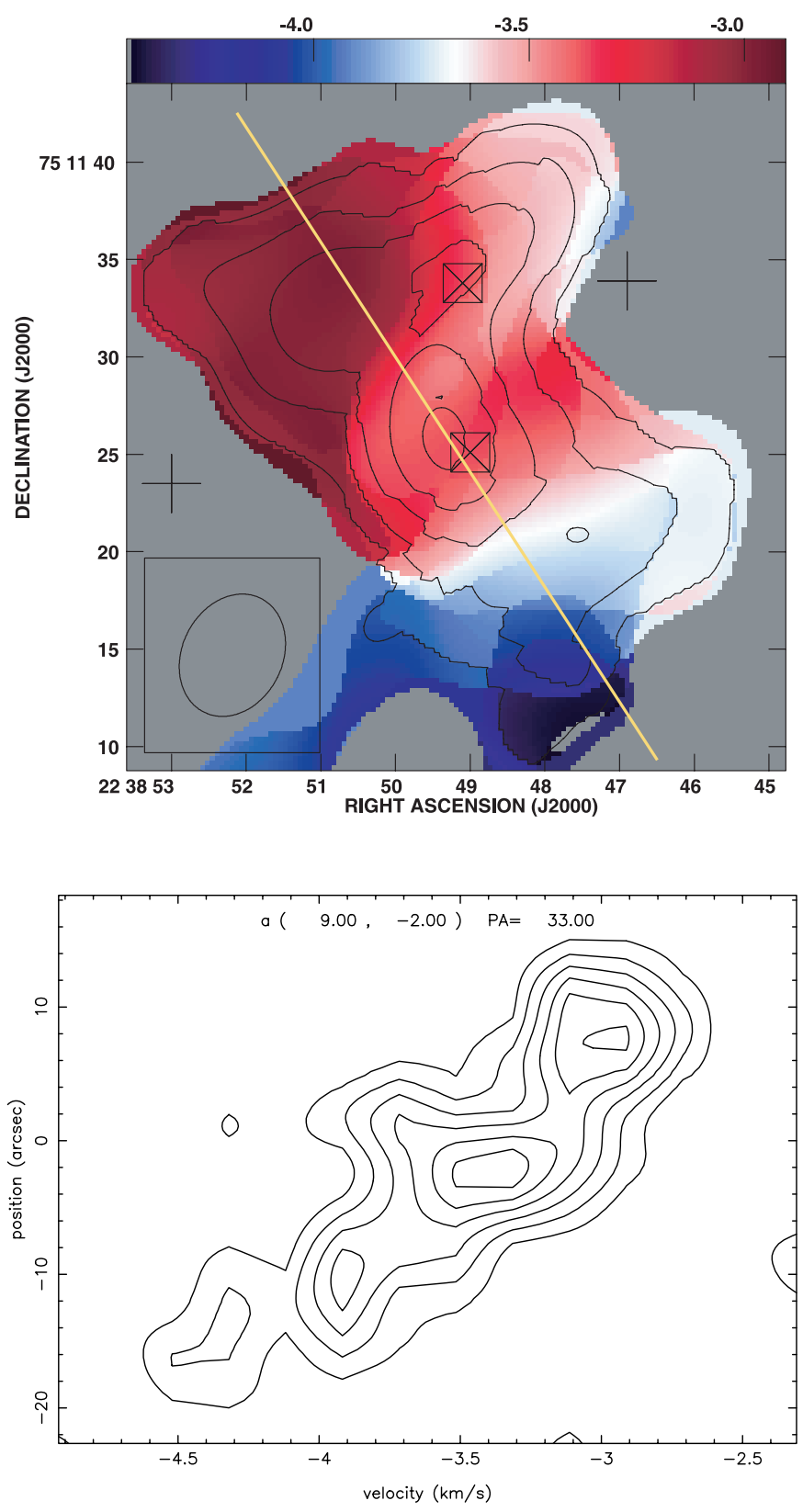

FIG. 12.-Top: Integrated intensity contours overlaid onto the mean centroid velocity image of the isolated component $(F=01-12)$ of $\mathrm{N}_{2} \mathrm{H}^{+} 1-0$ observed at the OVRO. The moments were calculated in channels with emission $>2 \sigma$. The contours begin at $3 \sigma$ and increase in steps of $3 \sigma$, where $1 \sigma$ is $1.5 \mathrm{~K} \mathrm{~km} \mathrm{~s}^{-1}$. Crosses denote the positions of IRS1 and IRS2, and the square-X symbols indicate the positions of SMA-N and SMA-S. The ellipse to the lower left represents the synthesized beam of the $\mathrm{N}_{2} \mathrm{H}^{+} 1-0$ observation. The overlaid line indicates the direction for the position-velocity diagram in the bottom panel. Bottom: Positionvelocity diagram of the isolated component along the cut that centers at the emission peak, $(\Delta \alpha, \Delta \delta)=\left(9^{\prime \prime},-2^{\prime \prime}\right)$ with P.A. $=33^{\circ}$. This cut is basically along the integrated intensity map elongated in the direction of northeast-southwest crossing the emission peak. For these figures, the isolated component has been shifted in velocity by $8.0 \mathrm{~km} \mathrm{~s}^{-1}$. The contours begin at $2 \sigma$ and increase in steps of $2 \sigma$, where $1 \sigma$ is $0.25 \mathrm{~K} \mathrm{beam}^{-1}$.

(Note, however, that the temperature there cannot be greater than $30 \mathrm{~K}$, because otherwise $\mathrm{CO}$ would evaporate off dust grains and reduce the $\mathrm{N}_{2} \mathrm{D}^{+}$abundance.)

Our high-resolution observations of L1251B show further examples of the chemical behavior expected in the presence of nearby protostellar heating. Once a protostar forms, the surrounding material is heated up to the $\mathrm{CO}$ evaporation temperature 


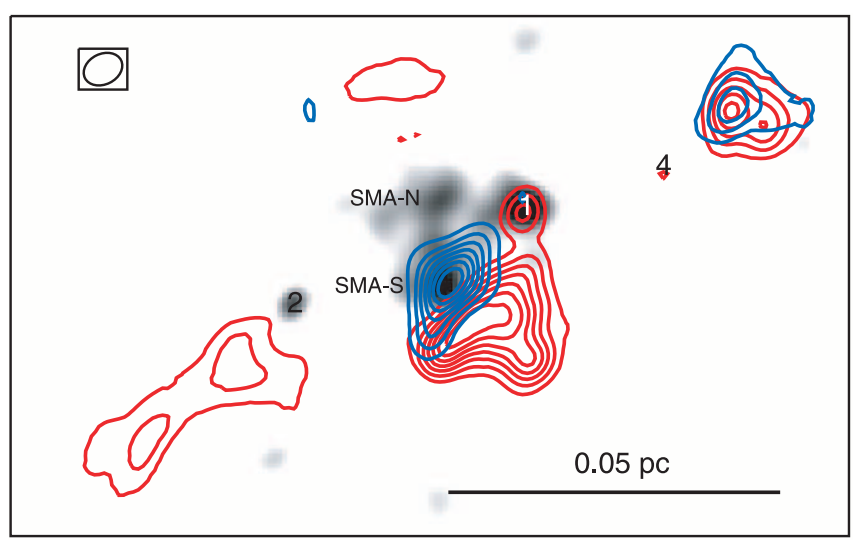

FIG. 13.- Integrated intensities of red- and blueshifted emission of ${ }^{12} \mathrm{CO} 2-1$ overlaid onto the $1.3 \mathrm{~mm}$ continuum emission image observed at the SMA. Red and blue contours denote intensities calculated over "blue" and "red" velocity ranges, respectively. The same velocity ranges as in Fig. 5 were used. Numbers indicate positions of IRS1, IRS2, and IRS4. Two starless condensations are named SMA-N and SMA-S. The ellipse to the upper left denotes the synthesized beam of the $\mathrm{CO}$ $2-1$ observation. The contours begin at $4 \sigma$ and increase in steps of $4 \sigma$, where $1 \sigma=2.14 \mathrm{~K} \mathrm{~km} \mathrm{~s}^{-1}$. The gray scale range is $0.01-0.0275 \mathrm{Jy} \mathrm{beam}^{-1}$.

( $\sim 25 \mathrm{~K}$ in the case of bare $\mathrm{SiO}_{2}$ dust grains). The desorbed $\mathrm{CO}$ destroys $\mathrm{N}_{2} \mathrm{H}^{+}$, producing an $\mathrm{N}_{2} \mathrm{H}^{+}$emission "hole" around the central heating source, which is potentially observable with interferometers. Such holes may explain the lack of $\mathrm{N}_{2} \mathrm{H}^{+}$emission coincident with IRS1 and IRS2, as seen in Figures $7 a$ and $8 a$. In contrast, the abundances of other molecules, such as CS, $\mathrm{H}_{2} \mathrm{CO}$, and $\mathrm{HCO}^{+}$, can be enhanced due to protostellar heating resulting in the desorption of $\mathrm{CO}$ or the desorption of themselves off of dust grain mantles (Lee et al. 2004) as seen in Figure $7 b$. In L1251B, IRS1 is luminous enough to evaporate $\mathrm{CO}$ in its inner envelope. Similar $\mathrm{N}_{2} \mathrm{H}^{+}$emission holes have been seen toward NGC 1333 IRAS 4A (Di Francesco et al. 2001) and L483 (Jørgensen 2004).

Figure 18 shows the results of a chemical evolution model made specifically for L1251B to quantify the observed line emission distributions. For this model, we used the chemo-dynamical model developed by Lee et al. (2004). We updated the chemical network to include more recent results on the $\mathrm{N}_{2} \mathrm{H}^{+}$chemistry, however, including a new binding energy of $\mathrm{N}_{2}$, which is the same as that of $\mathrm{CO}$ (Öberg et al. 2005), and new rates of dissociative recombination of $\mathrm{N}_{2} \mathrm{H}^{+}$with electrons (Geppert et al. 2004). We also increased the initial abundance of sulfur by a factor of 3 to fit the CS 5-4 line profile in Figure 10a. The initial abundances of other species are the same as those in Table 3 of Lee et al. (2004). For a dynamical model, we adopted the best-fit Shu inside-out collapse model to the dust continuum emission as described in $\S$ 4.1. Although L1251B has several sources, only one internal luminosity source at the center of a spherically symmetric envelope was assumed in the model. Since IRS1 is the dominant luminosity source by a factor of $\sim 10$, however (see Paper I), this model is a reasonable first approximation, especially for interpreting the single-dish observations. For this model, the infall rate from the disk to central protostar was tuned appropriately to match the luminosity calculated by observations at the given timescale (Young \& Evans 2005). In addition, the interstellar radiation field was assumed to be attenuated to $G_{0}=0.3$ for consistency with the dust continuum modeling. An outflow was not included.

We do not compare this chemical model with interferometric observations quantitatively since the density profile assumed in the one-dimensional model is not appropriate for the high-resolution observations that reveal multiple sources. The chemical distribution close to the central source, however, is most sensitive to the temperature environment, so we can look for the effects of temperature increases around IRS1 on the chemical abundances. As seen in Figure 18, the model predicts a $\mathrm{CO}$ evaporation radius (which accordingly is also the $\mathrm{N}_{2} \mathrm{H}^{+}$depletion radius) of $\sim 0.007 \mathrm{pc}$ $\left(\sim 4^{\prime \prime}\right)$, i.e., similar to the observed radius of the $\mathrm{N}_{2} \mathrm{H}^{+}$hole at a $2 \sigma$ level of integrated intensity (see Fig. 7a). Furthermore, the model predicts an $\mathrm{H}_{2} \mathrm{CO}$ abundance peak at a radius of $\sim 0.003 \mathrm{pc}$ $\left(\sim 2^{\prime \prime}\right)$, similar to the radius of the $\mathrm{H}_{2} \mathrm{CO}$ emission at the $2 \sigma$ level of the integrated intensity (Fig. $7 b$ ). A second $\mathrm{H}_{2} \mathrm{CO}$ abundance peak seen in Figure 18 caused by $\mathrm{CO}$ evaporation does not affect significantly the $\mathrm{H}_{2} \mathrm{CO}$ emission distribution, likely because of the lower density and temperature of material at $\sim 0.006 \mathrm{pc}$ relative to material at $\sim 0.003 \mathrm{pc}$ associated with the inner abundance peak. Therefore, this simple model is in good agreement with our observations close to the central source, where infall is kinematically dominant, and chemistry mainly depends on the evaporation of molecules from grain surfaces by heating.

Near IRS4, the associated $\mathrm{H}_{2} \mathrm{CO}$ emission may not be due to an increased abundance due to localized dust grain heating. That source may not be luminous enough to heat grains above the $\mathrm{H}_{2} \mathrm{CO}$ evaporation temperature at a projected distance of $600 \mathrm{AU}$. The coincidence of the maxima of the integrated intensity of $\mathrm{H}_{2} \mathrm{CO}$ $3_{12}-2_{11}$ (see Fig. $7 b$ ) to one of that of $\mathrm{HCO}^{+} 1-0$ (see Fig. 7a) suggests a possible origin from shocked outflow material. Note that the $\mathrm{H}_{2} \mathrm{CO}$ emission close to IRS4 lies at redshifted velocities of -1.5 to $-3 \mathrm{~km} \mathrm{~s}^{-1}$, and lies spatially at a full synthesized beam width $\left(2^{\prime \prime}\right)$ northeast of IRS4.

\section{DISCUSSION}

\section{1. $L 1251 B$}

L1251B harbors a small group of starless and protostellar objects. Three of the latter are classified as Class $0 /$ I candidates, which are associated with dense envelopes. The observations of molecular lines around L1251B have revealed active processes, which are either dynamical (infall, rotation, and outflow) or chemical (depletion and enhancement).

The $1.3 \mathrm{~mm}$ dust continuum observations of L1251B with the SMA revealed two condensations (SMA-N and SMA-S) that are unassociated with any detected near-infrared source (nNote that SMA-S may contain a newly formed protostellar object if an outflow originates from it; see Figs. 14 and 15, and $\S$ 4.3). Table 4 shows masses along the line of sight toward IRS1, IRS2, SMA-N, and SMA-S, determined using the peak intensities of the $1.3 \mathrm{~mm}$ image and assuming a dust temperature of $20 \mathrm{~K}$. We determined masses along the lines of sight rather than total masses from the respective fluxes because the extent of each was difficult to define with certainty in the crowded field, especially for SMA-N and SMA-S. Furthermore, any differences in mass along the line of sight could be more easily discerned with a single sampling. Table 4 shows that the peak intensities are quite similar (i.e., within $50 \%$ ), suggesting quite similar masses along the line of sight if the temperatures are common. If we assume for SMA-N and SMA-S a lower temperature of $10 \mathrm{~K}$, which is more commonly adopted for starless cores, their masses along the line of sight are greater than those for $20 \mathrm{~K}$ (and IRS1 and IRS2) by a factor of $\sim 3$.

Given their designations as protostars, the fluxes of IRS1 and IRS2 likely include emission from their respective disk components. In particular, IRS2 is not resolved at the $\sim 4^{\prime \prime}$ resolution of the data, which is equivalent to a $\sim 1200 \mathrm{AU}$ linear distance at the $300 \mathrm{pc}$ distance of L1251B. Since these sources are also 


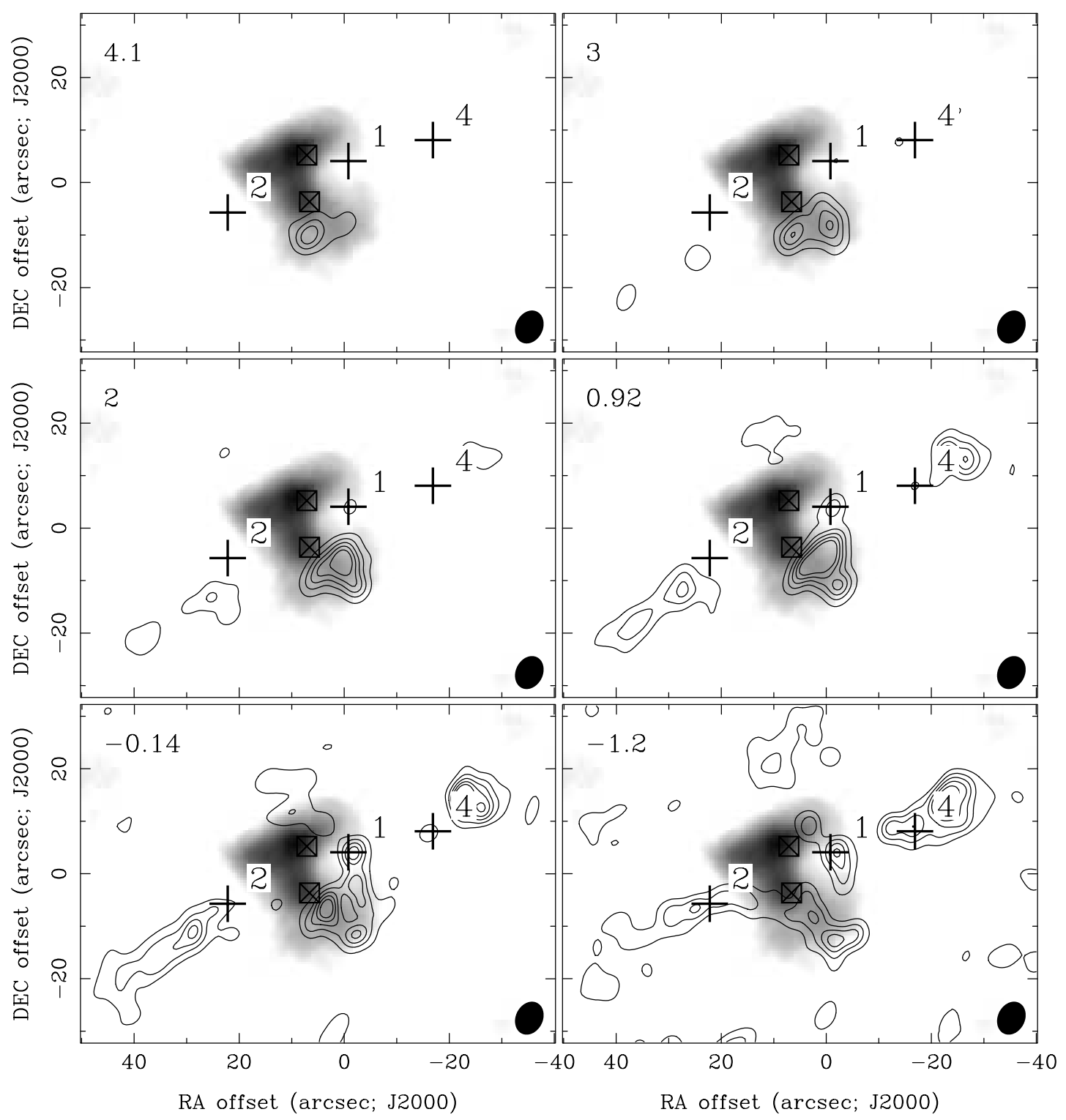

FIG. 14. - Channel map of redshifted emission of ${ }^{12} \mathrm{CO} 2-1$ observed at the SMA overlaid onto the integrated intensity image of $\mathrm{N}_{2} \mathrm{H}^{+} 1-0$ observed at the OVRO. The contours begin at $6 \sigma$ and increase in steps of $9 \sigma$, where $1 \sigma$ is $0.36 \mathrm{~K}$. The gray scale range is $1.5-15 \mathrm{~K} \mathrm{~km} \mathrm{~s}^{-1}$. Two square-X symbols indicate the positions of SMA-N and SMA-S, and crosses denote the positions of IRS1, IRS2, and IRS4. The ellipse to the lower right in each panel denotes the synthesized beam of the CO $2-1$ observation.

highly embedded, however, the $1.3 \mathrm{~mm}$ fluxes also likely contain emission from their inner envelopes. In contrast, the fluxes of SMA-N and SMA-S likely include emission only from envelopes since these sources are not seen if lower spatial frequencies (i.e., $<11 \mathrm{k} \lambda$ ) are excluded, as discussed in Paper I. To distinguish the disks of IRS1 and IRS2 (as well as a possible disk of SMA-S) associated with outflows ( $§ 4.3$ ), observations with higher resolution are needed. Note that $1.3 \mathrm{~mm}$ continuum emission is not detected toward IRS4, suggesting that it is not associated with any disk or inner envelope that could be detected at our sensitivity and resolution.

All objects in L1251B possibly form by fragmentation during gravitational collapse (Boss 1997; Machida et al. 2005). Turbulent fragmentation seems unlikely since the lines observed toward L1251B are not so broad as to be considered dominated by turbulence. For example, tracers of very dense gas such as $\mathrm{DCO}^{+}$ $3-2, \mathrm{~N}_{2} \mathrm{H}^{+} 1-0$, and $\mathrm{H}^{13} \mathrm{CO}^{+} 1-0$ have line widths of $\sim 1 \mathrm{~km} \mathrm{~s}^{-1}$, consistent with the infall velocity at $n \sim 10^{6} \mathrm{~cm}^{-3}$ in the best-fit inside-out collapse model of L1251B. In addition, CS 5-4, which traces much higher densities $\left(n \geq 5 \times 10^{6} \mathrm{~cm}^{-3}\right)$, shows the broadest line width among all the high-density tracers we observed toward L1251B, i.e., $\sim 2.6 \mathrm{~km} \mathrm{~s}^{-1}$. Indeed, our insideout collapse model shows an infall velocity at $5 \times 10^{6} \mathrm{~cm}^{-3}$ of $\sim 2 \mathrm{~km} \mathrm{~s}^{-1}$. In addition, the infall velocity profile of this model fits the observed CS 5-4 and $\mathrm{HCO}^{+} 3-2$ reasonably well, with constant turbulent widths of 0.6 and $0.25 \mathrm{~km} \mathrm{~s}^{-1}$ at radii less than and greater than the infall radius, respectively (see Fig. 10a). The velocity dispersions implied from such widths are not much greater than the thermal velocity dispersion before collapse. (The dotted line in Fig. 10 presents the case without the infall velocity structure.) Therefore, the dynamics of L1251B seem dominated by gravitational collapse, combined with rotation and outflows, rather than turbulent motions.

\subsection{The East Core}

The east core seen in Figure 1 at $850 \mu \mathrm{m}$ and in various molecular lines has a radius of about $0.1 \mathrm{pc}$. The $850 \mu \mathrm{m}$ emission shows several subcores inside a larger structure traced by molecular 


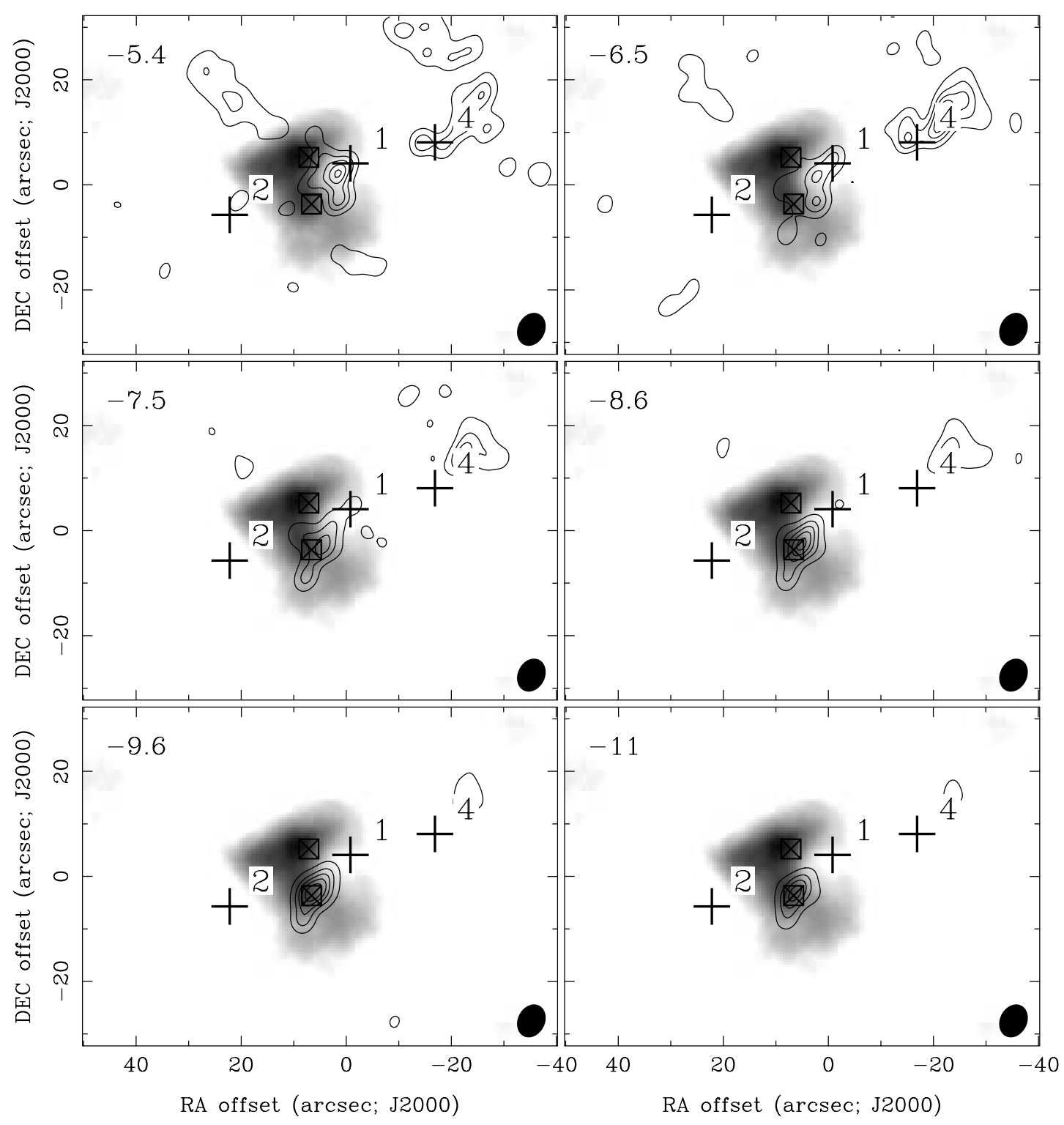

FIG. 15. - Channel map of blueshifted emission of ${ }^{12} \mathrm{CO} 2-1$ observed at the SMA overlaid onto the integrated intensity image of $\mathrm{N}_{2} \mathrm{H}^{+} 1-0$ observed at the OVRO. The contour levels and gray scale are the same as in Fig. 14.

emission. This difference may be related to a bias toward imaging smaller scale emission in the continuum data and related to high optical depths in the line data. For the continuum data, the James Clerk Maxwell Telescope SCUBA observations were made by chopping onto the sky, effectively filtering out emission on angular scales greater than the $\sim 120^{\prime \prime}$ chop throw (see Di Francesco et al. 2007), i.e., those traced by the molecular line data. In addition, the $850 \mu \mathrm{m}$ continuum emission is very likely optically thin, tracing quite dense material within a molecular core. For the line data, the molecular emission (see Fig. 1) may have high optical depths and the subcores may not be visible, despite the similarities between the densities these particular lines trace and the mean density of the subcores, i.e., $\sim 10^{5} \mathrm{~cm}^{-3}$. Differences in the positions of maximum integrated intensities between these lines may also be due to their relative (but high) optical depths. (To determine whether the $\sim 3 \times$ higher resolution of the $850 \mu \mathrm{m}$ map relative to the molecular line maps in Fig. 1 contributed to the different appearance of the continuum and line maxima, we smoothed the $850 \mu \mathrm{m}$ map to a resolution similar to that of the line maps $\left[60^{\prime \prime}\right]$. The smoothed $850 \mu \mathrm{m}$ map, how- ever, still shows an emission hole near the maxima of the line maps.)

The low optical depth of the $850 \mu \mathrm{m}$ emission suggests that the much stronger continuum peak toward L1251B $\left(\sim 1 \mathrm{Jy} \mathrm{beam}^{-1}\right)$ relative to the east core $\left(\sim 0.2 \mathrm{Jy} \mathrm{beam}^{-1}\right)$ is indicative of a larger column density toward L1251B, even if L1251B has been heated by internal sources. (Note that the $850 \mu \mathrm{m}$ emission peaks close to SMA-S, not toward IRS1, as seen in Fig. 6 of Paper I.) The $850 \mu \mathrm{m}$ fluxes of L1251B and the whole east core are similar, suggesting that their masses are also similar if their temperatures and dust opacities are themselves similar, i.e., $\sim 4 \mathrm{Jy}$ and $\sim 2 M_{\odot}$ if $T=20 \mathrm{~K}$ and $\kappa_{1.3 \mathrm{~mm}}=0.02 \mathrm{~cm}^{2} \mathrm{~g}^{-1}$ (Krügel \& Siebenmorgen 1994). Since L1251B contains several YSOs but the east core does not, it is likely that L1251B is warmer than the east core, however. Continuum observations at multiple wavelengths and modeling are needed to determine better relative values of dust temperature.

Table 5 lists the integrated intensities of molecular lines observed toward the centers of L1251B and the east core. To probe for any relative chemical evolution in the two cores, we compare 


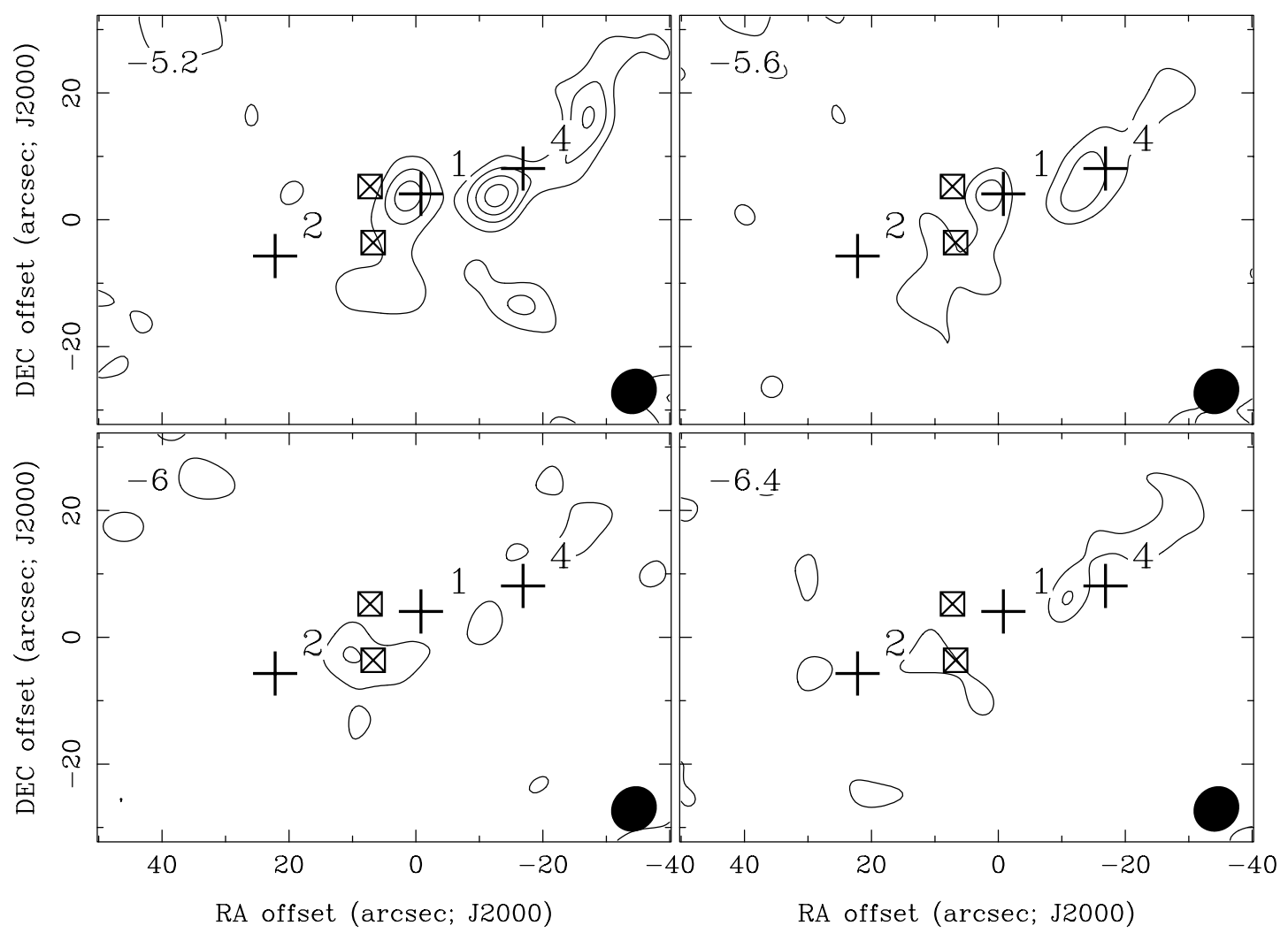

FIG. 16. - Channel map of blueshifted emission of $\mathrm{HCO}^{+} 1-0$ observed at the OVRO. Symbols are the same as in Fig. 14 and 15 . The contours begin at $2 \sigma$ and increase in steps of $2 \sigma$, where $1 \sigma=0.22 \mathrm{~K}$.

the relative integrated intensities of the 1-0 transitions of $\mathrm{H}^{13} \mathrm{CO}^{+} 1-0$ and $\mathrm{N}_{2} \mathrm{H}^{+}$, molecules found to be abundant respectively at earlier and later times in models of chemical evolution (e.g., Lee et al. 2003 and references therein). $\mathrm{For}^{13} \mathrm{CO}^{+}$ $1-0$, the integrated intensities of $\mathrm{L} 1251 \mathrm{~B}$ and the east core are similar (i.e., $\sim 1.3$ and $\sim 1.1 \mathrm{~K} \mathrm{~km} \mathrm{~s}^{-1}$, respectively). For $\mathrm{N}_{2} \mathrm{H}^{+}$, however, the integrated intensity toward L1251B is larger than that toward the east core by a factor of 2 (i.e., $\sim 4$ vs. $\sim 2 \mathrm{~K} \mathrm{~km} \mathrm{~s}^{-1}$, respectively). The relative integrated intensity ratio between these

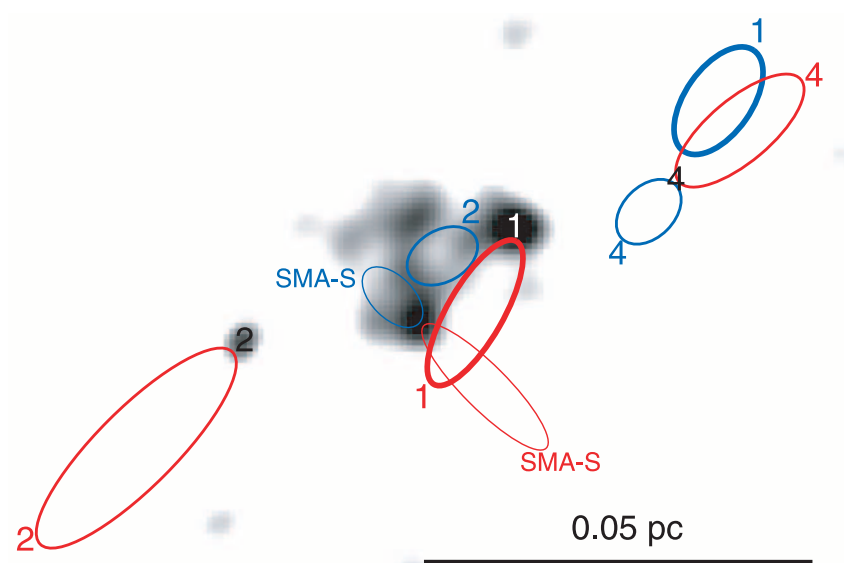

Fig. 17.- Possible explanation of the origin of each outflow. The gray image is the same as that in Fig. 13. Black and white numbers denote the positions of IRS1, IRS2, and IRS4. Red and blue lobes describe the red and blue components each outflow identified in the channel maps of $\mathrm{CO} 2-1$ and $\mathrm{HCO}^{+} 1-0$ observed at the SMA and the OVRO, respectively. Red and blue numbers and texts indicate the red- and blueshifted lobes originated from their corresponding sources.

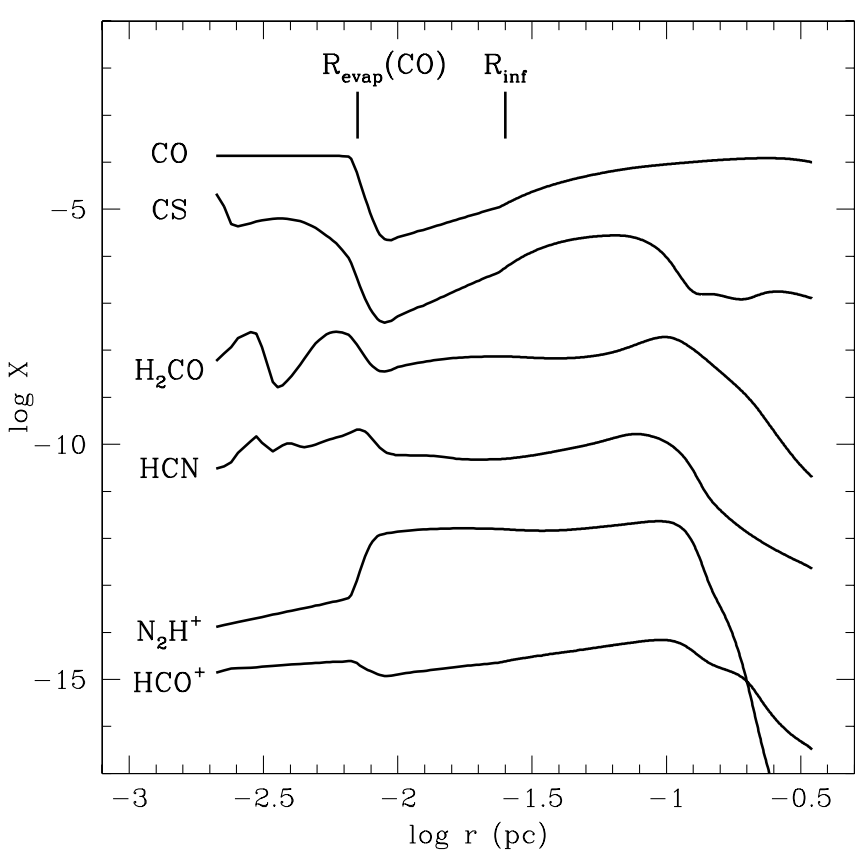

FIG. 18.-Abundance profiles of various molecules observed for this study. The profiles have been calculated using an evolutionary chemical model (Lee et al. 2004) defined on the basis of the physical parameters of L1251B. Two vertical lines indicate the infall radius of the inside-out collapse and the radius where $\mathrm{CO}$ starts to evaporate. Profiles are shifted up and down for easier comparisons, so the ordinate gives correct abundance of only $\mathrm{CO}$. Abundances of $\mathrm{CS}$ and $\mathrm{H}_{2} \mathrm{CO}$ are shifted up by 3.0 and 0.2 orders of magnitude respectively, while those of $\mathrm{HCN}$, $\mathrm{N}_{2} \mathrm{H}^{+}$, and $\mathrm{HCO}^{+}$are shifted down by 1.0, 2.5, and 6.0 orders of magnitude, respectively. 
TABLE 4

SMA 1.3 mm Peak Intensities and Masses

\begin{tabular}{|c|c|c|}
\hline Source & $\begin{array}{l}\text { Peak Intensity, } I(\lambda)^{\mathrm{a}} \\
\quad\left(\mathrm{Jy} \mathrm{beam}^{-1}\right)\end{array}$ & $\begin{array}{c}\text { Mass }^{\mathrm{b}} \\
\left(M_{\odot} \text { beam }^{-1}\right)\end{array}$ \\
\hline IRS1 & 0.035 & 0.031 \\
\hline IRS2 & 0.023 & 0.020 \\
\hline SMA-N .................................. & 0.023 & $0.020(0.055)^{\mathrm{c}}$ \\
\hline SMA-S & 0.029 & $0.026(0.070)^{\mathrm{c}}$ \\
\hline
\end{tabular}

${ }^{\mathrm{a}}$ Uncertainties on the peak intensities are $4 \mathrm{mJy}_{\text {beam }}{ }^{-1}$.

b Masses have been calculated based on the equation Mass $\left(M_{\odot}\right.$ beam $\left.^{-1}\right)=$ $\left[I(\lambda) d^{2}\right] /\left[\kappa_{\lambda} B_{\lambda}\left(T_{\text {dust }}\right)\right]=0.88 I(\lambda)\left(\mathrm{Jy} \mathrm{beam}^{-1}\right)$ at $d=300 \mathrm{pc}$ and $\lambda=1.3 \mathrm{~mm}$, which is derived from the assumptions of (1) the $1.3 \mathrm{~mm}$ opacity $\left(\kappa_{\lambda}\right)$ of $2 \times$ $10^{-2} \mathrm{~cm}^{2} \mathrm{~g}^{-1}$ (Krügel \& Siebenmorgen 1994) and (2) the dust temperature $\left(T_{\text {dust }}\right)$ of $20 \mathrm{~K}$ for the Planck function, $B_{\lambda}$.

${ }^{c}$ The mass if $T_{\text {dust }}=10 \mathrm{~K}$.

lines, i.e., $\sim 6$ for L1251B and $\sim 3$ for the east core, may be due to the relative chemical evolution, with L1251B being more chemically evolved. (Note that the difference in $\mathrm{N}_{2} \mathrm{H}^{+}$abundance might be greater than the difference in the $\mathrm{N}_{2} \mathrm{H}^{+} 1-0$ intensity, due to the higher optical depth in L1251B.) Such differences in chemical evolution may result from differences in density, since the chemical timescale shortens at a higher density. In addition, $\mathrm{H}^{13} \mathrm{CO}^{+}$ and its isotopologue $\mathrm{HCO}^{+}$deplete significantly more than $\mathrm{N}_{2} \mathrm{H}^{+}$ in cold, dense environments such as in L1251B (except in the immediate vicinities of protostellar objects). Therefore, the higher ratio between the $\mathrm{N}_{2} \mathrm{H}^{+}$and $\mathrm{H}^{13} \mathrm{CO}^{+}$intensities in $\mathrm{L} 1251 \mathrm{~B}$ than in the east core (Fig. 1) may be caused by a denser environment in L1251B.

Various lines toward the east core suggest infall motions. Therefore, the east core may be in earlier states of dynamical and chemical evolution than those of L1251B, and may harbor star formation in the future.

\section{SUMMARY}

L1251E, the densest $\mathrm{C}^{18} \mathrm{O}$ core of L1251 (Sato et al. 1994), has been observed at various molecular line transitions with singledish telescopes, and $\mathrm{L} 1251 \mathrm{~B}$, the protostellar group in the $\mathrm{C}^{18} \mathrm{O}$ core, has been observed with millimeter interferometers. We have compared these molecular line data to continuum data presented in Paper I to study this region of L1251 more comprehensively. Our results include:

1. L1251E contains at least two cores, one coincident with L1251B and an east core detected from $850 \mu \mathrm{m}$ continuum emission and various emission lines. The integrated intensities of these lines show primary maxima that are coincident with L1251B and other, secondary maxima associated with the east core which are non-coincident. No embedded sources have been detected in the Spitzer IRAC or MIPS bands toward the east core (Paper I), suggesting that it is starless. Asymmetric profiles with stronger blue peaks have been detected toward the eastern core, however, that are suggestive of infall motions. The east core appears less dense and less chemically evolved than L1251B.

2. The large-scale outflow associated with L1251B has been mapped in CO 2-1 with the CSO. Red and blue components of the outflow overlap around the brightest protostar, IRS1, and the near-infrared bipolar nebula source, IRS2. The CO 2-1 and ${ }^{13} \mathrm{CO} 2-1$ maps observed with the SMA resolve the outflow into a few components.

3. $\mathrm{HCO}^{+} 3-2$ line emission observed with the CSO across L1251B shows widespread line asymmetry that suggests infall
TABLE 5

InTEGRated INTENSITIES OF Molecular Lines

\begin{tabular}{|c|c|c|}
\hline Line & L1251B & East Core \\
\hline $\mathrm{HCO}^{+} 1-0 \ldots \ldots \ldots \ldots . . . .$. & 6.6 & 7.6 \\
\hline $\mathrm{H}^{13} \mathrm{CO}^{+} 1-0 \ldots \ldots \ldots \ldots \ldots$ & 1.3 & 1.1 \\
\hline CS $2-1 \ldots \ldots \ldots \ldots \ldots \ldots$ & 3.2 & 2.8 \\
\hline 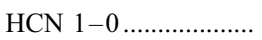 & $4.2(4)^{\mathrm{a}}$ & $3.0(0.85)^{\mathrm{a}}$ \\
\hline $\mathrm{N}_{2} \mathrm{H}^{+} 1-0 \ldots \ldots \ldots \ldots$ & $7.8(2.2)^{\mathrm{a}}$ & $3.8(1.7)^{\mathrm{a}}$ \\
\hline
\end{tabular}

Note.-In units of $\mathrm{K} \mathrm{km} \mathrm{s}^{-1}$.

${ }^{\mathrm{a}}$ The optical depth of the main component obtained by the hyperfine structure fit.

motions toward the central group, with evidence for rotational or outflow motions from modifications of the line profiles at many off-center locations.

4. On a large scale, L1251B has a velocity gradient that is half the magnitude of that in the starless east core and is opposite in direction. Previous low-resolution observations averaged the velocity gradients of both, causing rotational motions in the region to be misinterpreted.

5. $\mathrm{HCO}^{+} 1-0$ line emission observed with the OVRO MMA is centered at IRS1 and distributed linearly along the northwestsoutheast direction of the larger scale outflow. In contrast, the $\mathrm{N}_{2} \mathrm{H}^{+} 1-0$ line emission also observed with the OVRO MMA is distributed between IRS1 and IRS2 (like the position of maximum brightness of the IRAM $1.3 \mathrm{~mm}$ continuum emission) and extended perpendicular to the outflow, i.e., along the southwestnortheast direction. A position-velocity diagram of $\mathrm{N}_{2} \mathrm{H}^{+} 1-0$ indicates very fast rotation in this flattened envelope, i.e., $\Omega \sim$ $10^{-12} / \cos (i) \mathrm{s}^{-1}$, where $i$ is the inclination of the rotational axis from the plane of sky.

6. The maxima of $\mathrm{N}_{2} \mathrm{H}^{+} 1-0$ and $\mathrm{N}_{2} \mathrm{D}^{+} 3-2$ integrated intensity observed with the OVRO MMA and SMA are offset from locations of maximum continuum brightness from the two starless objects, which were newly detected at $1.3 \mathrm{~mm}$ with the SMA (Paper I). This offset may be due to reduction of $\mathrm{N}_{2} \mathrm{H}^{+}$and $\mathrm{N}_{2} \mathrm{D}^{+}$ abundances at very high densities.

7. OVRO MMA and SMA observations reveal a lack of $\mathrm{N}_{2} \mathrm{H}^{+}$emission at IRS1 (i.e., a "hole") but $\mathrm{C}^{18} \mathrm{O}$ and the $\mathrm{H}_{2} \mathrm{CO}$ emission is coincident with IRS1. These results are consistent with a chemo-dynamical evolution model where a central protostar heats up surrounding material. In this model, $\mathrm{CO}$ and $\mathrm{H}_{2} \mathrm{CO}$ are abundant close to the protostar where they are released into the gas phase from grain mantles, but $\mathrm{N}_{2} \mathrm{H}^{+}$has had its abundance sharply reduced since it is depleted by reactions with gas-phase CO.

Support for this study was provided by NASA through Hubble Fellowship grant HST-HF-01187 awarded by the Space Telescope Science Institute, which is operated by the Association of Universities for Research in Astronomy, Inc., for NASA, under contract NAS 5-26555. This work was also supported by NSF grant AST-0307350 to the University of Texas at Austin and by the state of Texas. We are very grateful to an anonymous referee for valuable comments. We thank Jonathan Williams for allowing us to use his unpublished molecular line data, and we also thank Chad Young for his help for the dust continuum modeling of L1251B. We are also very grateful to Anneila Sargent for providing us the opportunity to observe L1251B with the OVRO MMA. 
Aikawa, Y., Ohashi, N., Inutsuka, S.-I., Herbst, E., \& Takakuwa, S. 2001, ApJ, 552,639

Anglada, G., Sepúlveda, I., \& Gómez, J. F. 1997, A\&AS, 121, 255

Bergin, E. A., \& Langer, W. D. 1997, ApJ, 486, 316

Boss, A. P. 1997, ApJ, 483, 309

Caselli, P., Benson, P. J., Myers, P. C., \& Tafalla, M. 2002, ApJ, 572, 238

Caselli, P., Myers, P. C., \& Thaddeus, P. 1995, ApJ, 455, L77

Choi, M., Evans, N. J., II, Gregersen, E. M., \& Wang, Y. 1995, ApJ, 448, 742

Di Francesco, J., André, P., \& Myers, P. C. 2004, ApJ, 617, 425

Di Francesco, J., Evans, N. J., II, Caselli, P., Myers, P. C., Aikawa, Y., \& Tafalla, M. 2007, in Protostars and Planets V, ed. B. Reipurth, D. Jewitt, \& K. Kiel, (Tucson: Univ. Arizona), 17

Di Francesco, J., Johnstone, D., Kirk, H. M., MacKenzie, T., \& Ledwosinska, E. 2007, ApJS, submitted

Di Francesco, J., Myers, P. C., Wilner, D. J., Ohashi, N., \& Mardones, D. 2001, ApJ, 562, 770

Doty, S. D., \& Neufeld, D. A. 1997, ApJ, 489, 122

Doty, S. D., van Dishoeck, E. F., van der Tak, F. F. S., \& Boonman, A. M. S. 2002, A\&A, 389, 446

Evans, N. J., II, Rawlings, J. M. C., Shirley, Y., \& Mundy, L. G. 2001, ApJ, 557,193

Geppert, W. D., et al. 2004, ApJ, 609, 459

Goodman, A. A., Benson, P. J., Fuller, G. A., \& Myers, P. 1993, ApJ, 406, 528

Gregersen, E. M., Evans, N. J., II, Mardones, D. M., \& Myers, P. C. 2000, ApJ, 533,440

Hogerheijde, M. R., \& van der Tak, F. F. S. 2000, A\&A, 362, 697

Jørgensen, J. K. 2004, A\&A, 424, 589

Krügel, E., \& Siebenmorgen, R. 1994, A\&A, 288, 929

Kun, M., \& Prusti, T. 1993, A\&A, 272, 235

Lada, C. J., \& Lada, E. A. 2003, ARA\&A, 41, 57

Lee, J.-E., Bergin, E. A., \& Evans, N. J., II. 2004, ApJ, 617, 360

Lee, J.-E., Evans, N. J., II, Shirley, Y. L., \& Tatematsu, K. 2003, ApJ, 583, 789

Lee, J.-E., et al. 2006, ApJ, 648, 491

Machida, M. N., Matsumoto, T., Hanawa, T., \& Tomisaka, K. 2005, MNRAS, 362,382
Mardones, D., Myers, P. C., Tafalla, M., Wilner, D. J., Bachiller, R., \& Garay, G. 1997, ApJ, 489, 719

Morata, O., Estalella, R., López, R., \& Planesas, P. 1997, MNRAS, 292, 120

Myers, P. C., Evans, N. J., II, \& Ohashi, N. 2000, in Protostars and Planets IV, ed. V. Mannings, A. P. Boss, \& S. S. Russell (Tucson: Univ. Arizona), 217

Öberg, K. I., van Broekhuizen, F., Fraser, H. J., Bisschop, S. E., van Dishoeck, E. F., \& Schlemmer, S. 2005, ApJ, 621, L33

Pagani, L., Pardo, J.-R., Apponi, A. J., Bacmann, A., \& Cabrit, S. 2005, A\&A, 429, 181

Pickett, H. M., Poynter, R. L., Cohen, E. A., Delitsky, M. L., Pearson, J. C., \& Muller, H. S. P. 1998, J. Quant. Spectrosc. Radiat. Transfer, 60, 883

Rawlings, J. M. C., Redman, M. P., Keto, E., \& Williams, D. A. 2004, MNRAS, 351, 1054

Rawlings, J. M. C., \& Yates, J. A. 2001, MNRAS, 326, 1423

Rebull, L. M., et al. 2007, ApJS, 171, 447

Sato, F., \& Fukui, Y. 1989, ApJ, 343, 773

Sato, F., Mizuno, A., Nagahama, T., Onishi, T., Yonekura, Y., \& Fukui, Y. 1994, ApJ, 435, 279

Shu, F. H. 1977, ApJ, 214, 488

Snell, R. L., Loren, R. B., \& Plambeck, R. L. 1980, ApJ, 239, L17

Terebey, S., Shu, F. H., \& Cassen, P. 1984, ApJ, 286, 529

Tóth, L. V., \& Walmsley, C. M. 1996, A\&A, 311, 981

Walker, C. K., Narayanan, G., \& Boss, A. P. 1994, ApJ, 431, 767

Ward-Thompson, D., \& Buckley, H. D. 2001, MNRAS, 327, 955

Williams, J. P, Bergin, E. A., Caselli, P., Myers, P. C., \& Plume, R. 1998, ApJ, 503,689

Wilson, T. L., \& Rood, R. T. 1994, ARA\&A, 32, 191

Young, C. H., \& Evans, N. J., II. 2005, ApJ, 627, 293

Young, C. H., Shirley, Y. L., Evans, N. J., II, \& Rawlings, J. M. C. 2003, ApJS, 145,111

Young, K. E., Lee, J.-E., Evans, N. J., II, Goldsmith, P. F., \& Doty, S. D. 2004, ApJ, 614, 252

Zhou, S. 1995, ApJ, 442, 685

Zhou, S., Evans, N. J., II, Koempe, C., \& Walmsley, C. M. 1993, ApJ, 404, 232 